\title{
Consistent set of band parameters for the group-III nitrides AIN, GaN, and InN
}

\author{
Patrick Rinke, ${ }^{1, *}$ M. Winkelnkemper, ${ }^{1,2}$ A. Qteish, ${ }^{3}$ D. Bimberg, ${ }^{2}$ J. Neugebauer, ${ }^{4}$ and M. Scheffler ${ }^{1}$ \\ ${ }^{1}$ Fritz-Haber-Institut der Max-Planck-Gesellschaft, Faradayweg 4-6, D-14195 Berlin, Germany \\ ${ }^{2}$ Institut für Festkörperphysik, Technische Universität Berlin, Hardenbergstraße 36, D-10623 Berlin, Germany \\ ${ }^{3}$ Department of Physics, Yarmouk University, 21163-Irbid, Jordan \\ ${ }^{4}$ Department of Computational Materials Design, Max-Planck-Institut fur Eisenforschung, D-40237 Düsseldorf, Germany
}

(Received 26 October 2007; published 19 February 2008)

\begin{abstract}
We have derived consistent sets of band parameters (band gaps, crystal field splittings, band-gap deformation potentials, effective masses, and Luttinger and $E_{P}$ parameters) for $\mathrm{AlN}, \mathrm{GaN}$, and $\mathrm{InN}$ in the zinc-blende and wurtzite phases employing many-body perturbation theory in the $G_{0} W_{0}$ approximation. The $G_{0} W_{0}$ method has been combined with density-functional theory (DFT) calculations in the exact-exchange optimized effective potential approach to overcome the limitations of local-density or gradient-corrected DFT functionals. The band structures in the vicinity of the $\Gamma$ point have been used to directly parametrize a $4 \times 4 \mathbf{k} \cdot \mathbf{p}$ Hamiltonian to capture nonparabolicities in the conduction bands and the more complex valence-band structure of the wurtzite phases. We demonstrate that the band parameters derived in this fashion are in very good agreement with the available experimental data and provide reliable predictions for all parameters, which have not been determined experimentally so far.
\end{abstract}

DOI: $10.1103 /$ PhysRevB.77.075202

PACS number(s): 71.15.Mb, 71.20.Nr

\section{INTRODUCTION}

The group-III nitrides AlN, GaN, and InN and their alloys have become an important and versatile class of semiconductor materials, in particular, for use in optoelectronic devices and high-power microwave transistors. Current applications in solid state lighting (light emitting diodes and laser diodes) range from the visible spectrum ${ }^{1-4}$ to the deep ultraviolet, ${ }^{5,6}$ while future applications, e.g., chemical sensors ${ }^{7-9}$ or in quantum cryptography, ${ }^{10}$ are being explored.

For future progress in these research fields, reliable material parameters beyond the fundamental band gap, such as effective electron masses and valence-band (Luttinger or Luttinger-like) parameters, are needed to aid the interpretation of experimental observations and to simulate (hetero-) structures, e.g., optoelectronic devices. Material parameters can be derived from first-principles electronic-structure methods for bulk phases, but the size and complexity of structures required for device simulations currently far exceed the capabilities of first-principles electronic-structure tools. To bridge this gap, first-principles calculations can be used to parametrize simplified methods, such as the $\mathbf{k} \cdot \mathbf{p}$ method, ${ }^{11-14}$ the empirical tight-binding method, ${ }^{15-18}$ or the empirical pseudopotential method (EPM) ${ }^{19}$ which are applicable to large-scale heterostructures at reasonable computational expense.

In this paper, we use many-body perturbation theory in the $G_{0} W_{0}$ approximation ${ }^{20}$ - currently the method of choice for the description of quasiparticle band structures in solids $^{21-23}$-in combination with the $\mathbf{k} \cdot \mathbf{p}$ approach, ${ }^{11-14}$ to derive a consistent set of material parameters for the groupIII nitride system. The $\mathbf{k} \cdot \mathbf{p}$-Hamiltonian is parametrized to reproduce the $G_{0} W_{0}$ band structure in the vicinity of the $\Gamma$ point. Since the parameters of the $\mathbf{k} \cdot \mathbf{p}$ method are closely related or, in some cases, even identical to basic band parameters, many key band parameters can be directly obtained using this approach.
The $\mathbf{k} \cdot \mathbf{p}$-model Hamiltonian is typically parametrized for bulk structures and is then applicable to heterostructures with finite size (e.g., micro- and nanostructures) within the envelope-function scheme. ${ }^{24}$ Ideally, the parameters are determined entirely from consistent experimental inputs. For the group-III-nitrides, however, many of the key band parameters have not been conclusively determined until now, despite the extensive research effort in this field. ${ }^{25,26}$ In a comprehensive review, Vurgaftman and Meyer summarized the field of III-V semiconductors in 2001 and recommended up-to-date band parameters for all common compounds and their alloys including the nitrides. ${ }^{27}$ Only two years later, they realized that "it is striking how many of the nitride properties have already been superseded, not only quantitatively but qualitatively." ${ }^{26}$ They proceeded to "remedy that obsolescence, by providing a completely revised and updated description of the band parameters for nitride-containing semiconductors" in 2003. ${ }^{26}$ While this update includes evidence supporting a revision of the band gap of $\mathrm{InN}$ from its former value of $1.9 \mathrm{eV}$ to a significantly lower value around $0.7 \mathrm{eV}, 28-32$ they had to concede that in many cases experimental information on certain parameters was simply not available. ${ }^{26}$ This was mostly due to growth-related difficulties in producing high-quality samples for an unambiguous characterization. In the meantime, the quality of, e.g., wurtzite InN samples has greatly improved, ${ }^{25}$ and even the growth of the zinc-blende phase has advanced..$^{33}$ Nevertheless, many of the basic material properties of the group-III nitrides are still undetermined or, at least, controversial.

On the theoretical side, certain limitations of densityfunctional theory (DFT) in the local-density approximation (LDA) or generalized gradient approximation (GGA) currently the most widespread ab initio electronic-structure method for polyatomic systems - have hindered an unambiguous completion of the missing data. To overcome these deficiencies, we use $G_{0} W_{0}$ calculations based on DFT calculations in the exact-exchange optimized effective potential (OEPx) approach to determine the basic band parameters. 
We have previously shown that the $\mathrm{OEPx}+G_{0} W_{0}$ approach provides an accurate description of the quasiparticle band structure for $\mathrm{GaN}$, InN, and II-VI compounds. ${ }^{23,34,35}$ The quasiparticle band structure in the vicinity of the $\Gamma$ point is then used to parametrize a $4 \times 4 \mathbf{k} \cdot \mathbf{p}$ Hamiltonian to determine band-dispersion parameters, such as effective masses, Luttinger parameters, etc. This allows us to take the nonparabolicity of the conduction band, which is particularly pronounced in $\mathrm{InN},{ }^{29,36}$ and the more complex valence-band structure of the wurtzite phases into account properly.

This paper is organized as follows: In Sec. II, we briefly introduce the $G_{0} W_{0}$ approach and its application to the group-III nitrides, followed by a discussion of certain key parameters of the quasiparticle band structure, such as the fundamental band gaps (and their dependence on the unitcell volume) and the crystal-field splitting energies (Sec. III). In Sec. IV, we present our recommendations for the banddispersion parameters $(\mathbf{k} \cdot \mathbf{p}$ parameters) of the wurtzite and zinc-blende phases of AlN, GaN, and InN. A detailed discussion of the parameter sets is given in Sec. IV B together with a comparison to experimental values and parameter sets obtained by other theoretical approaches. Our conclusions are given in Sec. V.

\section{QUASIPARTICLE ENERGY CALCULATIONS}

\section{A. $G W$ based on exact-exchange density functional theory}

The root of the deficiencies in LDA and GGA for describing spectroscopic properties such as the quasiparticle band structure can be found in a combination of different factors. LDA and GGA are approximate (jellium-based) exchangecorrelation functionals, which suffer from an incomplete cancellation of artificial self-interaction and lack the discontinuity of the exchange-correlation potential with respect to the number of electrons. As a consequence, the Kohn-Sham single-particle eigenvalues cannot be rigorously interpreted as the quasiparticle band structure as measured by direct and inverse photoemission. This becomes most apparent for the band gap, which is severely underestimated by the KohnSham eigenvalue difference in LDA and GGA. For InN, this even results in an overlap between the conduction and the valence bands and thus an effectively metallic state, as displayed in Fig. 1. It goes without mentioning that a $\mathbf{k} \cdot \mathbf{p}$ parametrization derived from this LDA band structure would not appropriately reflect the properties of bulk InN.

Many-body perturbation theory in the $G W$ approach $^{20}$ presents a quasiparticle theory that overcomes the deficiencies of LDA and GGA and provides a suitable description of the quasiparticle band structure of weakly correlated solids, such as $\mathrm{AlN}, \mathrm{GaN}$, and $\mathrm{InN}^{21-23}$ Most commonly, the Green's function $G_{0}$ and the screened potential $W_{0}$ required in the $G W$ approach (henceforth denoted $G_{0} W_{0}$ ) are calculated from a set of DFT Kohn-Sham single-particle energies and wave functions. The DFT ground state calculation is typically carried out in the LDA or GGA, and the quasiparticle corrections to the Kohn-Sham eigenvalues are calculated in first order perturbation theory (LDA/GGA $\left.+G_{0} W_{0}\right)$ without resorting to self-consistency in $G$ and $W .{ }^{37}$

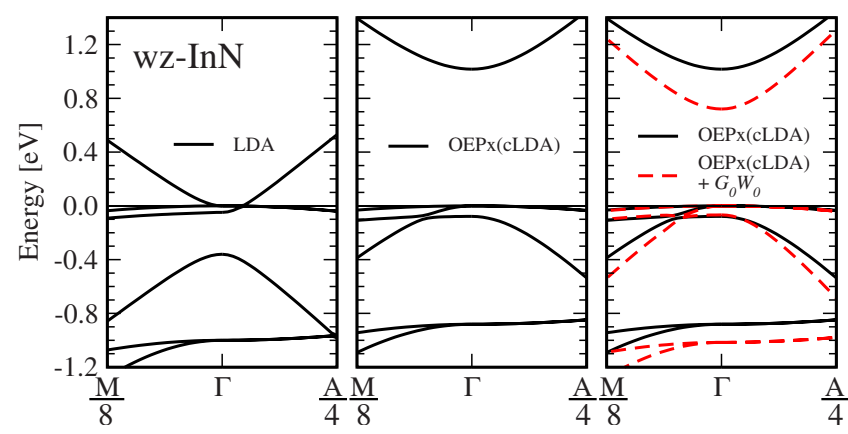

FIG. 1. (Color online) LDA Kohn-Sham calculations incorrectly predict wurtzite InN to be a metal with the wrong band ordering at the $\Gamma$ point. In OEPx(cLDA), the band gap opens and InN correctly becomes a semiconductor, thus providing a more suitable starting point for subsequent quasiparticle energy calculations in the $G_{0} W_{0}$ approximation.

While the LDA $+G_{0} W_{0}$ approach is now almost routinely applied to bulk materials, ${ }^{21-23} G_{0} W_{0}$ calculations for GaN and $\mathrm{InN}$ have been hampered by the deficiencies of the LDA. For zinc-blende $\mathrm{GaN}$ the LDA $+G_{0} W_{0}$ band gap of $2.88 \mathrm{eV}$ (Refs. 38 and 39) is still too low compared to the experimental $3.3 \mathrm{eV},{ }^{40-42}$ while for InN the LDA predicts a metallic ground state with incorrect band ordering. A single $G_{0} W_{0}$ iteration proves not to be sufficient to restore a proper semiconducting state and only opens the band gap to $0.02-0.05 \mathrm{eV}, 43,44$ which are still far from the experimental value of $\sim 0.7 \mathrm{eV} .^{28-30}$

Here, we apply the $G_{0} W_{0}$ approach to DFT calculations in the OEPx or OEPx(cLDA) if LDA correlation is included. In contrast to LDA and GGA, the OEPx approach is fully selfinteraction-free and correctly predicts $\mathrm{InN}$ to be semiconducting with the right band ordering in the wurtzite phase, ${ }^{34,45}$ as Fig. 1 demonstrates. For II-VI compounds, $\mathrm{GaN}$ and $\mathrm{ScN}$, we have previously illustrated the mechanism behind this band-gap opening in the OEPx approach, ${ }^{23,34,35,46}$ which brings the OEPx Kohn-Sham band gap much closer to the experimental one. Combining OEPx with $G_{0} W_{0}$ (Ref. 47) in this fashion yields band gaps for II-VI compounds, Ge, $\mathrm{GaN}$, and $\mathrm{ScN}$, in very good agreement with experiment. ${ }^{23,34,35,46}$ For wurtzite $\mathrm{InN}$, the band gap of $0.7 \mathrm{eV}$ and the nonparabolicity of the conduction band $(\mathrm{CB})^{34}$ (shown in Fig. 1) strongly support the recent experimental findings. ${ }^{28-30,36,48}$ In addition, we have shown that the source for the startling wide interval of experimentally observed band gaps can be consistently explained by the Burstein-Moss effect (apparent band-gap increase with increasing electron concentration in the conduction band) by extending our calculations to finite carrier concentrations. ${ }^{34}$

\section{B. Computational parameters}

The LDA and OEPx calculations in the present work were performed with the plane-wave, pseudopotential code S/PHI/NX, ${ }^{50}$ while for the $G_{0} W_{0}$ calculations we have employed the $G_{0} W_{0}$ space-time method $^{51}$ in the gwst implementation. ${ }^{52-54}$ Local LDA correlation is added in all OEPx calculations. Here, we follow the parametrization of 
TABLE I. Experimental lattice parameters $\left(a_{0}, c_{0}\right.$, and $\left.u\right)$ adopted in this work (see text) for zinc blende (zb) and wurtzite (wz) AlN, GaN, and InN (Ref. 49).

\begin{tabular}{lcccc}
\hline \hline & $\begin{array}{c}a_{0} \\
(\AA)\end{array}$ & $\begin{array}{c}c_{0} \\
(\AA)\end{array}$ & $c_{0} / a_{0}$ & $u$ \\
\hline zb-AlN & 4.370 & & & \\
zb-GaN & 4.500 & & & \\
zb-InN & 4.980 & & & \\
wz-AlN & 3.110 & 4.980 & 1.6013 & 0.382 \\
wz-GaN & 3.190 & 5.189 & 1.6266 & 0.377 \\
wz-InN & 3.540 & 5.706 & 1.6120 & 0.380 \\
\hline \hline
\end{tabular}

Perdew and Zunger ${ }^{55}$ for the correlation energy density of the homogeneous electron gas based on the data of Ceperley and Alder. ${ }^{56}$ This combination will be denoted as OEPX(cLDA) in the following. Consistent pseudopotentials were used throughout, i.e., exact-exchange pseudopotentials ${ }^{57}$ for the OEPx(cLDA) and LDA ones for the LDA calculations. The cation $d$ electrons were included explicitly. ${ }^{23,45}$ For additional technical details and convergence parameters, we refer to previous work. ${ }^{23,34}$

\section{Lattice parameters}

All calculations are carried out at the experimental lattice constants reported in Table I and not at the $a b$ initio ones to avoid artificial strain effects in the derived parameter sets. For an $a b$ initio determination of the lattice constants consistent with the $G_{0} W_{0}$ calculations, the crystal structure would have to be optimized within the $G_{0} W_{0}$ formalism, too. However, $G_{0} W_{0}$ total energy calculations for realistic systems have, to our knowledge, not been performed yet, and the quality of the $G_{0} W_{0}$ total energy for bulk semiconductors has not been assessed so far. ${ }^{58}$ The alternative $a b$ initio choices, LDA and OEPx(cLDA), give different lattice constants ${ }^{59}$ and would thus introduce uncontrollable variations in the calculated band parameters that would aggravate a direct comparison.
The thermodynamically stable phase of $\mathrm{InN}$ at the usual growth conditions is the wurtzite phase. Reports of a successful growth of the zinc-blende phase have been scarce. Recently, high-quality films of zb-InN grown on indium oxide have been obtained by Lozano et $a .^{33}$ We adopt their lattice constant of $4.98 \AA,{ }^{33}$ which is in good agreement with previous reports of $4.98 \AA,{ }^{60} 4.986 \AA$, ${ }^{61}$ and $5.04 \AA$ (Ref. 62) for wz-InN grown on different substrates. For zb-AlN, reports of successful growth are even scarcer. Petrov et al. first grew AlN in the zinc-blende phase and reported a lattice constant of $a_{0}=4.38 \AA .{ }^{63}$ This was later refined by Thompson et al. to $a=4.37 \AA,{ }^{64}$ which is the value we adopt in this work. For zb-GaN, we follow the work of Lei et al. ${ }^{65,66}$ and choose $a_{0}=4.50 \AA$.

Although wurtzite is the phase predominantly grown for InN, reported values for the structural parameters still scatter appreciably. ${ }^{67}$ In order to determine the effect of the lattice constants on the band gap $\left(E_{g}\right)$ and the crystal-field splitting $\left(\Delta_{\mathrm{CR}}\right)$, we have explored the range between the maximum and minimum values of $a_{0}$ and $c_{0} / a_{0}$ reported in Ref. 67 by performing OEPx $(\mathrm{cLDA})+G_{0} W_{0}$ calculations at the values listed in Table II. Since $u$ remains undetermined in Ref. 67, we have optimized it in the LDA. Neither $u, E_{g}$, nor $\Delta_{\mathrm{CR}}$ depends sensitively on the lattice constants in this regime, and we have therefore adopted the mean values of $a_{0}$ $=3.54 \AA, c_{0}=5.706 \AA\left(c_{0} / a_{0}=1.612\right)$, and $u=0.380$ (the LDA-optimized value) for the remainder of this paper.

For wz-AlN and wz-GaN, the lattice constants are more established. ${ }^{26,68}$ For wz-AlN, we adopt Schulz and Thiermann's values of $a=3.110 \AA, c=4.980 \AA$, and $u=0.382,{ }^{69}$ which are close to those reported by Yim et al. ${ }^{70}$ Schulz and Thiermann also provided a value for the internal parameter $u$, which is identical to the one we obtain by relaxing $u$ in the LDA at the experimental $a_{0}$ and $c_{0}$ parameters. The same is true for wz-GaN. Schulz and Thiermann's values of $a$ $=3.190 \AA$ and $c=5.189 \AA$ (Ref. 69) are close to those first reported by Maruska and Tietjen, ${ }^{71}$ but in addition offer a value of $u=0.377$, which corresponds to our LDA-relaxed value at the same lattice parameters. Note that the lattice parameters of wz-InN and wz-GaN have been refined compared to our recently published calculations. ${ }^{34}$ The influence of the adjustment on the different band parameters will be discussed where necessary.

TABLE II. Band gap $\left(E_{g}\right)$ and crystal-field splitting $\left(\Delta_{\mathrm{CR}}\right)$ for wurtzite InN in the range of the experimentally reported values of the structural parameters $a_{0}$ and $c_{0} / a_{0}$ (Ref. 67) and $u$ determined in LDA for $a_{0}=3.54 \AA$ and $c_{0} / a_{0}=1.612$.

\begin{tabular}{|c|c|c|c|c|c|c|}
\hline \multirow[b]{2}{*}{$\begin{array}{l}a_{0} \\
(\AA)\end{array}$} & \multirow[b]{2}{*}{$c_{0} / a_{0}$} & \multirow[b]{2}{*}{$u$} & \multicolumn{2}{|c|}{$\operatorname{OEPx}(\mathrm{cLDA})+G_{0} W_{0}$} & \multicolumn{2}{|c|}{$\operatorname{OEPx}(\mathrm{cLDA})$} \\
\hline & & & $\begin{array}{l}\Delta_{\mathrm{CR}} \\
(\mathrm{eV})\end{array}$ & $\begin{array}{c}E_{g} \\
(\mathrm{eV})\end{array}$ & $\begin{array}{l}\Delta_{\mathrm{CR}} \\
(\mathrm{eV})\end{array}$ & $\begin{array}{c}E_{g} \\
(\mathrm{eV})\end{array}$ \\
\hline 3.535 & 1.612 & 0.380 & 0.067 & 0.71 & 0.079 & 1.01 \\
\hline 3.540 & 1.612 & 0.380 & 0.066 & 0.69 & 0.079 & 1.00 \\
\hline 3.545 & 1.612 & 0.380 & 0.065 & 0.68 & 0.079 & 0.98 \\
\hline 3.540 & 1.611 & 0.380 & 0.065 & 0.70 & 0.078 & 1.00 \\
\hline 3.540 & 1.612 & 0.380 & 0.066 & 0.69 & 0.079 & 1.00 \\
\hline 3.540 & 1.613 & 0.380 & 0.068 & 0.69 & 0.081 & 0.99 \\
\hline
\end{tabular}


TABLE III. Band gaps $\left(E_{g}\right)$ and crystal-field splittings $\left(\Delta_{\mathrm{CR}}\right)$ (Ref. 83) for the wurtzite and zinc-blende phases of AlN, GaN, and InN. All values are given in eV.

\begin{tabular}{|c|c|c|c|c|c|}
\hline & \multirow[b]{2}{*}{ Parameter } & \multirow{2}{*}{$\begin{array}{l}\mathrm{OEPx}(\mathrm{cLDA})+G_{0} W_{0} \\
\quad(\text { this work })\end{array}$} & \multirow[b]{2}{*}{ Expt. } & LDA & $\operatorname{EPx}(\mathrm{cLDA})$ \\
\hline & & & & \multicolumn{2}{|c|}{ (this work, for comparison) } \\
\hline \multicolumn{6}{|c|}{ Wurtzite } \\
\hline \multirow[t]{2}{*}{ wZ-AlN } & $E_{g}$ & 6.47 & $6.0-6.3^{\mathrm{a}}$ & 4.29 & 5.73 \\
\hline & $\Delta_{\mathrm{CR}}$ & -0.295 & $-0.230^{\mathrm{b}}$ & -0.225 & -0.334 \\
\hline \multirow[t]{2}{*}{ wz-GaN } & $E_{g}$ & 3.24 & $3.5^{\mathrm{c}}$ & 1.78 & 3.15 \\
\hline & $\Delta_{\mathrm{CR}}$ & 0.034 & $0.009-0.038^{\mathrm{c}}$ & 0.049 & 0.002 \\
\hline \multirow[t]{2}{*}{ wZ-InN } & $E_{g}$ & 0.69 & $0.65-0.8^{\mathrm{d}}$ & & 1.00 \\
\hline & $\Delta_{\mathrm{CR}}$ & 0.066 & $0.019-0.024^{\mathrm{e}}$ & & 0.079 \\
\hline \multicolumn{6}{|c|}{ Zinc blende } \\
\hline \multirow[t]{2}{*}{ zb-AlN } & $E_{g}^{\Gamma-\Gamma}$ & 6.53 & & 4.29 & 5.77 \\
\hline & $E_{g}^{\stackrel{g}{\Gamma-X}}$ & 5.63 & $5.34^{\mathrm{f}}$ & 3.28 & 5.09 \\
\hline $\mathrm{zb}-\mathrm{GaN}$ & $E_{g}$ & 3.07 & $3.3^{\mathrm{g}}$ & 1.64 & 2.88 \\
\hline zb-InN & $E_{g}$ & 0.53 & $0.6^{\mathrm{h}}$ & & 0.81 \\
\hline
\end{tabular}

${ }^{\mathrm{a}}$ References 70 and 72-77.

${ }^{b}$ Reference 72.

${ }^{\mathrm{c} R e f e r e n c e} 26$ and references therein.

${ }^{\mathrm{d}}$ References 28-30 and 79-82.

${ }^{\mathrm{e}}$ Reference 82.

${ }^{\mathrm{f}}$ Reference 64.

geferences 40-42.

${ }^{\mathrm{h}}$ Reference 78.

\section{BAND GAPS, CRYSTAL-FIELD SPLITTINGS, AND BAND-GAP DEFORMATION POTENTIALS}

We will now discuss the quasiparticle band structure of $\mathrm{AlN}, \mathrm{GaN}$, and $\mathrm{InN}$ in their zinc-blende and wurtzite phases in terms of certain key band parameters such as the band gap $\left(E_{g}\right)$, the crystal-field splitting $\left(\Delta_{\mathrm{CR}}\right)$ in the wurtzite phase, and the band-gap volume deformation potentials $\alpha_{V}$. At the end of this section, we will draw a comparison between LDA and OEPx (cLDA) based $G_{0} W_{0}$ calculations for AlN.

\section{A. Band gaps}

The OEPx $(\mathrm{cLDA})+G_{0} W_{0}$ band gaps for the three materials and two phases are reported in Table III together with the LDA and OEPx(cLDA) values for comparison. For GaN and $\mathrm{InN}$, the $\mathrm{OEPx}(\mathrm{cLDA})+G_{0} W_{0}$ band gaps have been reported previously in Ref. 34. There, we have also argued that the wide interval of experimentally observed band gaps for $\mathrm{InN}$ can be consistently explained by the Burstein-Moss effect. The OEPx $(\mathrm{cLDA})+G_{0} W_{0}$ value of $0.69 \mathrm{eV}$ for wz-InN (Ref. 84) supports recent observations of a band gap at the lower end of the experimentally reported range. For zinc-blende $\mathrm{InN}$, which has been explored far less experimentally, our calculated band gap of $0.53 \mathrm{eV}$ also agrees very well with the recently measured (and Burstein-Moss corrected) $0.6 \mathrm{eV}^{78}$

For $\mathrm{GaN}$, the band gaps of both phases are well established experimentally, and our OEPx (cLDA $)+G_{0} W_{0}$ calculated values of $3.24 \mathrm{eV}$ (Ref. 85) and $3.07 \mathrm{eV}$ agree to within $0.3 \mathrm{eV}$.
For AlN experimental results for the band gap of the wurtzite phase scatter appreciably, whereas for zinc blende only one value has - to the best of our knowledge-been reported so far. Contrary to GaN, the OEPx(cLDA) $+G_{0} W_{0}$ gaps for AlN are larger than the experimentally reported values.

\section{B. Crystal-field splitting}

Experimental values for the crystal-field splitting of wz-GaN scatter between 0.009 and $0.038 \mathrm{eV}$ (Table III). The OEPx $($ cLDA $)+G_{0} W_{0}$ value of $0.033 \mathrm{eV}$ supports a crystalfield splitting within this range.

Theoretical $^{86}$ and experimental ${ }^{72}$ investigations of wzAlN agree upon the fact that the crystal-field splitting of AlN is negative. Our calculations also yield a negative value of $\Delta_{\mathrm{CR}}=-0.295 \mathrm{eV}$. This result supports a crystal-field splitting in AlN below $-0.2 \mathrm{eV}$, as reported by Chen et al. ${ }^{72}$ rather than a small negative value between -0.01 and -0.02 , as implied by the results of Freitas et al. ${ }^{87}$ For wz-InN, a crystal-field splitting between 0.019 and $0.024 \mathrm{eV}$ has been reported recently. ${ }^{82}$ This value is significantly smaller than the $\operatorname{OEPx}(\mathrm{cLDA})+G_{0} W_{0}$ value of $0.07 \mathrm{eV}$.

The crystal-field splitting is known to be sensitive to lattice deformations, such as changes in the $c_{0} / a_{0}$ ratio or the internal lattice parameter $u .{ }^{88-90}$ Therefore, the discrepancy between experiment and theory might stem from the uncertainties of the lattice parameters of wz-InN (see Sec. II C). However, varying the $c_{0} / a_{0}$ ratio or the unit-cell volume within the experimental range discussed in Sec. II C yields 
TABLE IV. Volume band-gap deformation potentials $\left(\alpha_{V}\right)$ for the wurtzite and zinc-blende phases of $\mathrm{AlN}, \mathrm{GaN}$, and InN. The volume deformation potentials can be transformed into pressure deformation potentials using the bulk moduli of the respective materials (see text). All values are given in $\mathrm{eV}$.

\begin{tabular}{|c|c|c|c|c|}
\hline & & \multirow{2}{*}{$\begin{array}{l}\mathrm{OEPx}(\mathrm{cLDA})+G_{0} W_{0} \\
\quad \text { (this work) }\end{array}$} & \multirow{2}{*}{$\frac{\text { LDA }}{\text { (this } \mathrm{w}}$} & \multirow{2}{*}{$\begin{array}{l}\text { OEPx(cLDA) } \\
\text { for comparison) }\end{array}$} \\
\hline & & & & \\
\hline \multicolumn{5}{|c|}{ Wurtzite } \\
\hline AlN & $\alpha_{V}^{\mathrm{wz}}$ & -9.8 & -8.8 & -8.9 \\
\hline $\mathrm{GaN}$ & $\alpha_{V}^{\mathrm{wz}}$ & -7.6 & -6.8 & -6.5 \\
\hline $\mathrm{InN}$ & $\alpha_{V}^{\mathrm{wZ}}$ & -4.2 & & -3.0 \\
\hline \multicolumn{5}{|c|}{ Zinc blende } \\
\hline \multirow[t]{2}{*}{ AlN } & $\alpha_{V}^{\mathrm{zb} \Gamma-\Gamma}$ & -10.0 & -9.1 & -9.1 \\
\hline & $\alpha_{V}^{\mathrm{zb} \Gamma-X}$ & -1.8 & -0.6 & -0.9 \\
\hline $\mathrm{GaN}$ & $\alpha_{\Gamma}^{\mathrm{zb}}$ & -7.3 & -6.4 & -6.1 \\
\hline $\mathrm{InN}$ & $\alpha_{V}^{\mathrm{zb}}$ & -3.8 & & -2.6 \\
\hline
\end{tabular}

values for $\Delta_{\mathrm{CR}}$, which are always larger than $0.06 \mathrm{eV}$ (Table II), leaving only the internal lattice parameter $u$ as a possible source of error. This parameter is - at least for $\mathrm{GaN}-$ known to have a large influence on the crystal-field splitting. ${ }^{90} \mathrm{Al}-$ though the LDA-optimized $u$ values are in very good agreement with experimental values for $\mathrm{GaN}$ and $\mathrm{AlN}$, an experimental confirmation of the $u$ parameter of $\mathrm{InN}$ is still pending. We therefore calculated the crystal-field splitting of wz-InN for different values of $u$ (and $a_{0}$ and $c_{0}$ fixed at the values listed in Table I) between 0.377 and 0.383 . Generally, $\Delta_{\mathrm{CR}}$ decreases with increasing $u$, but even for $u$ as large as 0.383 , the crystal-field splitting is still larger than $0.05 \mathrm{eV}$. The discrepancy between the experimental report and the OEPx $($ cLDA $)+G_{0} W_{0}$ calculations hence cannot be attributed to the uncertainties in the lattice parameters and has to remain unsettled for the time being.

\section{Band-gap deformation potentials}

For the hydrostatic band-gap deformation potentials, the band gaps have been calculated at different volumes $(V)$ between $\pm 2 \%$ around the equilibrium volume $V_{0}$. In the explored volume range, the band gaps vary linearly with $\ln \left(V / V_{0}\right)$. The linear coefficient is then taken as the hydrostatic volume deformation potential $\alpha_{V}$. The calculated bandgap deformation potentials are listed in Table IV for LDA, OEPx(cLDA), and OEPx(cLDA) $+G_{0} W_{0}$. We observe that for all compounds and phases the quasiparticle deformation potential is larger in magnitude than that of the DFT [LDA and $\operatorname{OEPx}(\mathrm{cLDA})]$ calculations; i.e., the band gaps vary stronger with volume deformations. Hydrostatic band gap deformation potentials obtained from $\mathrm{LDA}+U$ calculations have recently been reported for the wurtzite phases of $\mathrm{GaN}$ and $\mathrm{InN} .{ }^{91}$ Unlike in OEPx, where an improved description of the $p-d$ hybridization is achieved by the full removal of the self-interaction for all valence states, the $\mathrm{LDA}+U$ approach reduces the $p$ - $d$ repulsion by adding an on-site Coulomb correlation $U$ only to the semicore $d$ electrons.
With reference to the $\operatorname{OEPx}(\mathrm{cLDA})+G_{0} W_{0}$ deformation potentials, the $\mathrm{LDA}+U$ improves upon $\mathrm{LDA}$ for $\mathrm{GaN}$ $\left(\alpha_{V}^{\mathrm{LDA}+U}=-7.7 \mathrm{eV}\right)$ but worsens for $\operatorname{InN}\left(\alpha_{V}^{\mathrm{LDA}+U}\right.$ $=-3.1 \mathrm{eV}, \alpha_{V}^{\mathrm{LDA}}=-4.2 \mathrm{eV}$ in Ref. 91).

Experimentally, the band-gap deformation potential is usually measured as a function of the applied pressure, which aggravates a direct comparison to our calculated volume deformation potentials. However, since $B=-d P / d \ln V$, where $B$ is the bulk modulus and $P$ the pressure, the pressure deformation potential $\alpha_{P}$ can be expressed in terms of $\alpha_{V}$ according to $\alpha_{P}=-\alpha_{V} / B$.

Experimentally reported values for the bulk modulus of wz-GaN scatter between 1880 and 2450 kbar. $^{92-96}$ Using these values, our volume deformation potential of $\alpha_{V}=-7.6 \mathrm{eV}$ would translate into a pressure deformation potential in the range of 3.1-4.0 meV/kbar, which is comparable to the experimentally determined range of 3.7-4.7 meV/kbar. ${ }^{96-101}$ This large uncertainty has been partially ascribed to the low quality of earlier samples and substrate-induced strain effects. ${ }^{100}$ The fact that the pressure dependence of the band gap is sublinear (unlike the volume dependence) further questions the accuracy of linear or quadratic fits for the extraction of the deformation potentials in the experiments. 100

For wz-InN, experimentally reported values are sparse. Franssen et al. determined a hydrostatic pressure deformation potential of $2.2 \mathrm{meV} / \mathrm{kbar},{ }^{102}$ while $\mathrm{Li}$ et al. found $3.0 \mathrm{meV} / \mathrm{kbar}^{103}$ This range agrees with our theoretical one of $2.8-3.3 \mathrm{meV} / \mathrm{kbar}$, using for the conversion of volume to pressure deformation potentials the bulk modulus range of 1260-1480 kbar (Refs. 94 and 95) quoted in the literature.

For wz-AlN, we are only aware of one experimental study reporting a pressure deformation potential of $4.9 \mathrm{meV} / \mathrm{kbar}^{75}$ With experimental bulk moduli between 1850 and 2079 kbar (Refs. 104-106), the OEPx(cLDA) $+G_{0} W_{0}$ pressure deformation potential of wz-AlN would fall between 4.7 and $5.3 \mathrm{meV} / \mathrm{kbar}$, straddling the experimentally reported value.

To our knowledge, no experimental information on the deformation potential of zb-AlN and zb-InN are available. For $\mathrm{zb}-\mathrm{GaN}$, our computed volume deformation potential of $\alpha_{V}=-7.3 \mathrm{eV}$ translates to a pressure deformation potential range of 3.0-3.9 meV/kbar using the same bulk modulus range as for wz-GaN. This range is slightly below the experimentally reported range of 4.0-4.6 meV/kbar. ${ }^{101,107,108} \mathrm{Em}$ ploying a semiempirical approach to overcome the band-gap underestimation of the LDA [LDA-plus-correction (LDA $+C)$, see also the discussion in Sec. IV B 1], Wei and Zunger found volume deformation potentials of $-10.2 \mathrm{eV}$ [zb-AlN $(\Gamma-\Gamma)],-1.1 \mathrm{eV}$ [zb-AlN $(\Gamma-X)],-7.4 \mathrm{eV}(\mathrm{zb}-\mathrm{GaN})$, and $-3.7 \mathrm{eV}(\mathrm{zb}-\mathrm{InN}),{ }^{109}$ in good agreement with our full OEPx $($ cLDA $)+G_{0} W_{0}$ calculations (see Table IV).

\section{Comparison between LDA $+G_{0} W_{0}$ and OEPx(cLDA) $+G_{0} W_{0}$}

For the materials presented in this paper, a meaningful comparison between LDA and $\operatorname{OEPx}(\mathrm{cLDA})$ based $G_{0} W_{0}$ calculations can only be constructed for AlN for reasons 


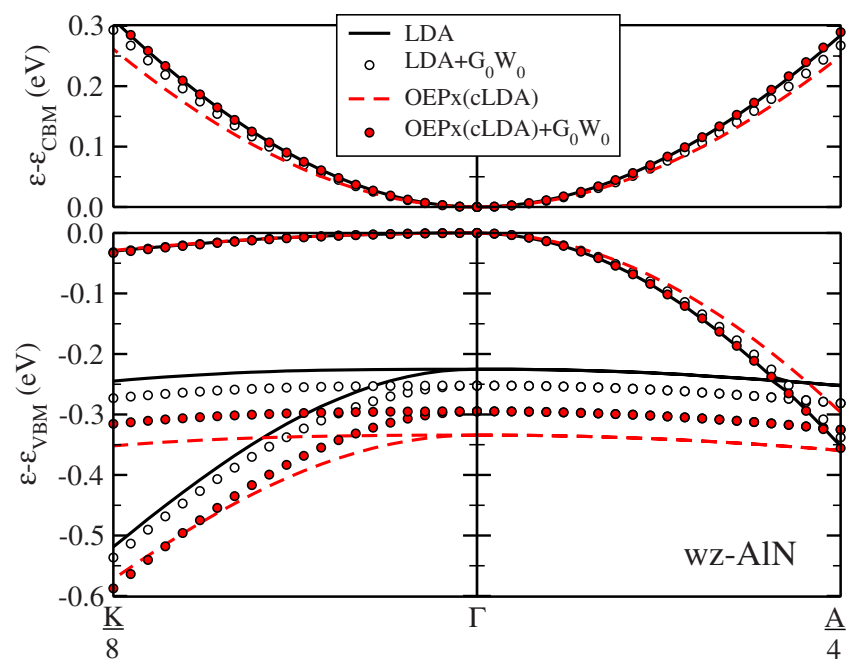

FIG. 2. (Color online) Comparison between LDA (solid lines), $\mathrm{LDA}+G_{0} W_{0}$ (open circles), OEPx(cLDA) (dashed lines), and $\operatorname{OEPx}(\mathrm{cLDA})+G_{0} W_{0}$ (solid circles) for wz-AlN. In both $G_{0} W_{0}$ calculations, bands are essentially shifted rigidly compared to the LDA, whereas OEPx(cLDA) yields bands with different dispersions.

given in Sec. II A. Figure 2 displays the band structure of WZ-AIN in the four approaches discussed in this paper. The "band-gap problem" has been eliminated from this comparison by aligning the conduction bands at the minimum of the lowest conduction band $\left(\epsilon_{\mathrm{CBM}}\right)$ and the valence bands at the maximum of the highest valence band $\left(\epsilon_{\mathrm{VBM}}\right)$. For this large gap material, three main conclusions can be drawn from Fig. 2. First, LDA and both $G_{0} W_{0}$ calculations yield very similar band dispersions. In other words, the $G_{0} W_{0}$ corrections to the LDA in the LDA $+G_{0} W_{0}$ approach are not $\mathbf{k}$ point dependent, shifting bands almost rigidly. A rigid shift between conduction and valence bands is frequently referred to as a "scissor operator." Figure 2, however, illustrates that this shift is not identical for all bands, which cannot be attributed to a single scissor operator. Second, the dispersion obtained in OEPX(cLDA) deviates from the other three approaches, which is consistent with the observation made for wz-InN in Fig. 1. We attribute this behavior to the approximate treatment of correlation in the OEPx(cLDA) approach and the fact that the band structure in OEPx(cLDA) is a Kohn-Sham and not a quasiparticle band structure. While the LDA benefits from a fortuitous error cancellation between the exchange and the correlation part, ${ }^{110}$ this is no longer the case once exchange is treated exactly in the OEPx(cLDA) scheme. Using a quasiparticle approach with a more sophisticated description of correlation, like the $G W$ method, then notably changes the dispersion of the OEPx(cLDA) bands. As we will demonstrate in the next section, this will lead to markedly different band parameters not only for the conduction but also for the valence bands (see Table VII). Against common belief, $\operatorname{OEPx}(\mathrm{cLDA})$ calculations without subsequent $G_{0} W_{0}$ calculations may therefore provide a distorted picture, and we would advise against deriving band parameters from OEPx or OEPx(cLDA) band structures alone. Third, unlike in the $\mathrm{LDA}+G_{0} W_{0}$ case, the $G_{0} W_{0}$ corrections to the OEPx(cLDA) starting point become $\mathbf{k}$ point dependent, a fact already observed for GaN and II-VI compounds. ${ }^{23}$ Most remarkably and in contrast to what we observe for $\mathrm{GaN}$ and $\mathrm{InN}$ (see Sec. IV B 2), the corrections are such that the band dispersion now agrees again with that obtained from the LDA and the LDA $+G_{0} W_{0}$ approach. Note also that both the band gap and the crystal-field splitting still differ slightly between $\mathrm{LDA}+G_{0} W_{0}$ and $\mathrm{OEPx}(\mathrm{cLDA})+G_{0} W_{0}$ for AlN $\left(E_{g}\right.$ : LDA $+G_{0} W_{0}: 5.95 \mathrm{eV}, \quad$ OEPx $(\mathrm{cLDA})+G_{0} W_{0}: \quad 6.47 \mathrm{eV}, \quad \Delta_{\mathrm{CR}}:$ $\mathrm{LDA}+G_{0} W_{0}: \quad-0.252 \mathrm{eV}, \quad$ and $\operatorname{OEPx}(\mathrm{cLDA})+G_{0} W_{0}$ : $-0.295 \mathrm{eV}$ ). Unlike for $\mathrm{GaN}$ and $\mathrm{InN}$, experimental uncertainties do, at present, not permit a rigorous assessment, which of the two $G_{0} W_{0}$ calculations provides a better description for AlN (see also Secs. III A and III B).

\section{BAND-DISPERSION PARAMETERS}

We will now turn our attention to band parameters that describe the band dispersion in the vicinity of the $\Gamma$ point: the effective masses, the Luttinger(-like) parameters, and the $E_{P}$ parameters. These parameters are obtained by means of the $\mathbf{k} \cdot \mathbf{p}$ method. The $\mathbf{k} \cdot \mathbf{p}$ method is a well-established approach that permits a description of semiconductor band structures in terms of parameters that can be accessed experimentally. Throughout this paper, we use a four-band $\mathbf{k} \cdot \mathbf{p}$ theory, which is typically used to describe direct-gap materials, mostly in its spin-polarized form as an eight-band $\mathbf{k} \cdot \mathbf{p}$ theory. The $\mathbf{k} \cdot \mathbf{p}$ Hamiltonian and all relevant formulas are given in Appendix A. The $\mathbf{k} \cdot \mathbf{p}$ method is a widely accepted technique for, e.g., the interpretation of experimental data $^{36,111}$ or modeling of semiconductor nanostructures and (opto-)electronic devices. ${ }^{12-14,112-115}$ Its accuracy, however, depends crucially on the quality of the input band parameters, such as effective electron masses, Luttinger parameters, etc., which have to be derived either experimentally or from band structure calculations. As alluded to in the Introduction, many important band parameters of the group-III nitrides $\mathrm{GaN}, \mathrm{InN}$, and $\mathrm{AlN}$ are still unknown. In particular, the band structure of $\mathrm{InN}$ is currently the subject of active research in both experiment and theory.

In this paper, we use the $\mathbf{k} \cdot \mathbf{p}$ method to derive banddispersion parameters from OEPx(cLDA $)+G_{0} W_{0}$ band structures. This approach has certain advantages over a simple parabolic approximation around the $\Gamma$ point. First, the $\mathbf{k} \cdot \mathbf{p}$ band structure is valid, not only directly at the $\Gamma$ point, but also in a certain $\mathbf{k}$ range around it. This allows us to extend the fit to larger k's and thereby increases the accuracy of the fitted parameters. Second, the $\mathbf{k} \cdot \mathbf{p}$ method is capable of describing nonparabolic bands, such as the $\mathrm{CB}$ of $\mathrm{InN},{ }^{34,36}$ and can therefore also be applied to accurately determine values for the effective electron masses and $E_{P}$ parameters in InN.

\section{A. Computational details}

For an accurate fit of the $\mathbf{k} \cdot \mathbf{p}$ parameters to the quasiparticle band structure, a small reciprocal lattice vector spacing is required. Since most $G W$ implementations evaluate the self-energy $\Sigma$ (the perturbation operator that links the KohnSham with the quasiparticle system) in reciprocal space, the 
matrix elements with respect to the Kohn-Sham wave functions $\left\langle\phi_{n \mathbf{q}}\left|\Sigma\left(\epsilon_{n \mathbf{q}}^{\mathrm{qp}}\right)\right| \phi_{n \mathbf{q}}\right\rangle$ required for the quasiparticle corrections are only available at the $\mathbf{k}$ points of the underlying $\mathbf{k}$ grid. A fine sampling of the $\Gamma$-point region would therefore be equivalent to using formidably large $\mathbf{k}$ grids in the computation. Most interpolation schemes that are frequently employed to calculate the quasiparticle corrections for arbitrary band structure points are to no avail in this case because they do not add new information to the fitting problem at hand. Existing schemes to directly compute the self-energy for band structure $\mathbf{q}$ points not contained in the $\mathbf{k}$ grid (see, for instance, Ref. 21 for an overview) are usually not implemented.

In the $G W$ space-time method, ${ }^{51}$ these problems are easily circumvented because the self-energy is computed in real space $\left[\Sigma_{\mathbf{R}}\left(\mathbf{r}, \mathbf{r}^{\prime} ; \epsilon\right)\right]$. By means of Fourier interpolation,

$$
\Sigma_{\mathbf{q}}\left(\mathbf{r}, \mathbf{r}^{\prime} ; \epsilon\right)=\sum_{\mathbf{R}} \Sigma_{\mathbf{R}}\left(\mathbf{r}, \mathbf{r}^{\prime} ; \epsilon\right) e^{-i \mathbf{q} \cdot \mathbf{R}},
$$

the self-energy operator can be calculated at arbitrary $\mathbf{q}$ points. ${ }^{52}$ The matrix elements $\left\langle\phi_{n \mathbf{q}}\left|\Sigma_{\mathbf{q}}(\epsilon)\right| \phi_{n \mathbf{q}}\right\rangle$ are then obtained by integration over $\mathbf{r}$ and $\mathbf{r}^{\prime}$. In this fashion, the relevant Brillouin zone regions for the band structure fitting can be calculated efficiently without compromising accuracy.

The $\mathbf{k} \cdot \mathbf{p}$ Hamiltonian and all parameter relations are given in Appendixes A and B. To determine the $\mathbf{k} \cdot \mathbf{p}$ Hamiltonian for a given band structure with band gap $E_{g}$ and crystal-field splitting $\Delta_{\mathrm{CR}}$, we fit the parameters $m_{e}^{i}, A_{i}, \gamma_{i}$, and $E_{P}^{i}$. This is achieved by least-squares-root fitting of the $\mathbf{k} \cdot \mathbf{p}$ band structure to the OEPx $(\mathrm{cLDA})+G_{0} W_{0}$ band structure in the vicinity of $\Gamma$. For the wurtzite phases, the directions $\Sigma, \Lambda, T$, and $\Delta$ have been included in the fit, represented by 22 equidistant $\mathbf{k}$ points from $\Gamma$ to $\frac{M}{8}\left(\frac{L}{8}, \frac{K}{8}\right)$ and 22 equidistant points from $\Gamma$ to $\frac{A}{4}$. For the zinc-blende phases, the directions $\Sigma, \Delta$, and $\Lambda$ have been included, each with 22 k points from $\Gamma$ to $\frac{K}{8}\left(\frac{X}{8}, \frac{L}{8}\right)$.

\section{B. Band parameters of GaN, AIN, and InN}

The parameters obtained by fitting to the OEPx(cLDA) $+G_{0} W_{0}$ band structures are listed in Table V (wz) and Table VI (zb). The resulting $\mathbf{k} \cdot \mathbf{p}$ band structures are plotted in Figs. 3 and 4 (black solid lines) together with the respective OEPx $(\mathrm{cLDA})+G_{0} W_{0}$ data (black circles). The excellent agreement of the $\mathbf{k} \cdot \mathbf{p}$ and $\mathrm{OEPx}(\mathrm{cLDA})+G_{0} W_{0}$ band structures illustrates that the band structures of the wurtzite and zinc-blende phases of all three materials are accurately described by the $\mathbf{k} \cdot \mathbf{p}$ method within the chosen $\mathbf{k}$ ranges. Additionally, the $\mathbf{k} \cdot \mathbf{p}$ band structures based on the parameters recommended by Vurgaftman and Meyer ${ }^{26,116}$ (VM'03) are shown (red dashed lines). As alluded to in the Introduction, their recommendations are based on available experimental data and selected theoretical values, representing the state-ofthe-art parameters up until the year of compilation (2003). We will also compare our results to more recent experimentally and theoretically derived parameters (see Table VII).

In the following, we will show that the parameters derived from the OEPx $(\mathrm{cLDA})+G_{0} W_{0}$ calculations match all avail-
TABLE V. Recommended band parameters for the wurtzite phases of $\mathrm{GaN}$, InN, and AlN derived from the OEPx(cLDA) $+G_{0} W_{0}$ band structures.

\begin{tabular}{lrrr}
\hline \hline Parameter & \multicolumn{1}{c}{ AlN } & GaN & \multicolumn{1}{c}{ InN } \\
\hline$m_{e}^{\|}$ & 0.322 & 0.186 & 0.065 \\
$m_{e}^{\perp}$ & 0.329 & 0.209 & 0.068 \\
$A_{1}$ & -3.991 & -5.947 & -15.803 \\
$A_{2}$ & -0.311 & -0.528 & -0.497 \\
$A_{3}$ & 3.671 & 5.414 & 15.251 \\
$A_{4}$ & -1.147 & -2.512 & -7.151 \\
$A_{5}$ & -1.329 & -2.510 & -7.060 \\
$A_{6}$ & -1.952 & -3.202 & -10.078 \\
$A_{7}(\mathrm{eV} \AA)$ & 0.026 & 0.046 & 0.175 \\
$E_{P}^{\|}(\mathrm{eV})$ & 16.972 & 17.292 & 8.742 \\
$E_{P}^{\perp}(\mathrm{eV})$ & 18.165 & 16.265 & 8.809 \\
\hline \hline
\end{tabular}

able experimental data to good accuracy. A comparison to parameters derived by other, theoretical or semiempirical, methods will be presented thereafter.

Before we proceed, however, we would like to emphasize two points regarding the relation between the VB parameters $A_{i}$ and the effective hole masses in wurtzite crystals: (i) Two different sets of equations, connecting the effective hole masses to the $A_{i}$ parameters, are used in the literature. Reference 117 lists both; one is labeled "near the band edge $(k$ $\rightarrow 0)$ " and the other "far away from the band edge ( $k$ is large)." The latter is widely used to calculate the effective hole masses. ${ }^{118-121}$ However, the experimentally relevant effective masses are those close to $\Gamma$. Thus, we use the near the band edge equations (see Appendix B) throughout this work. Quoted values differ from the original publications in cases where the original work uses the far away from the band edge equations. (ii) The Luttinger-like parameters, $A_{i}$, are independent of the spin-orbit and crystal-field interaction pa-

TABLE VI. Recommended band parameters for the zinc-blende phases of $\mathrm{GaN}$, InN, and AlN derived from the OEPx(cLDA) $+G_{0} W_{0}$ band structures. The effective hole masses for the $\mathrm{HH}$ and LH bands have been calculated from the Luttinger parameters (see Appendix B).

\begin{tabular}{lccc}
\hline \hline Parameter & AlN & GaN & InN \\
\hline$m_{e}(\Gamma)$ & 0.316 & 0.193 & 0.054 \\
$\gamma_{1}$ & 1.450 & 2.506 & 6.817 \\
$\gamma_{2}$ & 0.349 & 0.636 & 2.810 \\
$\gamma_{3}$ & 0.597 & 0.977 & 3.121 \\
$m_{\mathrm{hh}}^{[001]}$ & 1.330 & 0.810 & 0.835 \\
$m_{\mathrm{hh}}^{[110]}$ & 2.634 & 1.384 & 1.368 \\
$m_{\mathrm{hh}}^{[111]}$ & 3.912 & 1.812 & 1.738 \\
$m_{\mathrm{hh}}^{[001]}$ & 0.466 & 0.265 & 0.080 \\
$m_{\mathrm{lh}}^{[110]}$ & 0.397 & 0.233 & 0.078 \\
$m_{\mathrm{lh}}^{[111]}$ & 0.378 & 0.224 & 0.077 \\
$E_{P}(\mathrm{eV})$ & 23.844 & 16.861 & 11.373 \\
\hline \hline
\end{tabular}


(a) WZ-AlN

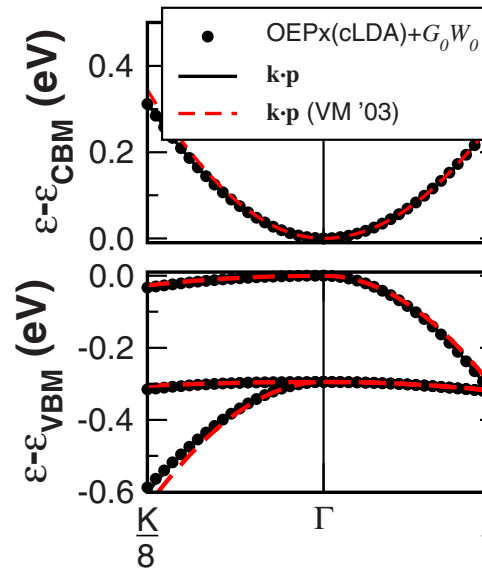

(b) wz-GaN

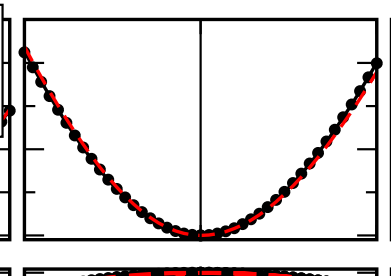

(c) $\mathrm{wZ}-\mathrm{InN}$

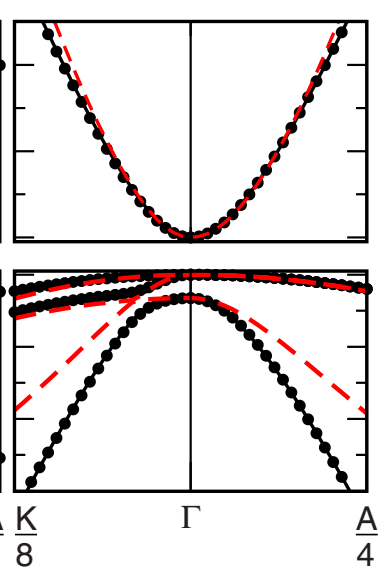

FIG. 3. (Color online) Band structure of wz-AlN (a), wz-GaN (b), and wz-InN (c) in the vicinity of $\Gamma$. The graphs show the $\operatorname{OEPx}(\mathrm{cLDA})+G_{0} W_{0}$ band structure (black circles), the corresponding $\mathbf{k} \cdot \mathbf{p}$ band structure (black solid lines), and the $\mathbf{k} \cdot \mathbf{p}$ band structure using the parameters recommended by Vurgaftman and Meyer (Refs. 26 and 116) (VM'03) (red dashed lines). rameters $\Delta_{\mathrm{SO}}$ and $\Delta_{\mathrm{CR}}$; the effective hole masses, however, differ for different $\Delta_{\mathrm{SO}}$ and $\Delta_{\mathrm{CR}}$ parameters. Only the $A$-band ( $C$-band in AlN) hole masses can be calculated from the Luttinger-like parameters alone. All other hole masses depend additionally on the choice of the spin-orbit and crystalfield splitting energies. ${ }^{117}$ Thus, effective $B$ - and $C$-band $(A$ and $B$-band in AlN) hole masses derived from different sets of Luttinger-like parameters are comparable, only if the same $\Delta_{\mathrm{SO}}$ and $\Delta_{\mathrm{CR}}$ values are assumed.

\section{Comparison to experimental values}

Experimentally, the band structure of a semiconductor is accessible only indirectly via band parameters such as $E_{g}$, $\Delta_{\mathrm{SO}}, \Delta_{\mathrm{CR}}$, and the effective masses. Angle resolved direct and inverse photoemission experiments, which would, in principle, directly probe the quasiparticle band structure, are not accurate enough yet to determine the band structure with sufficient accuracy.

The dispersion of the conduction band around the $\Gamma$ point depends only on the effective electron masses and $E_{P}$ parameters, which are accessible experimentally. The valence-band parameters, $A_{i}$, cannot be obtained directly experimentally but can be related to the effective hole masses (see Appendix B), which, in turn, can be measured.

The available experimental values for the wurtzite phases are listed in Table VII. For the thermodynamically metastable zinc-blende phases of GaN, AlN, and InN, hardly any experimental reports on their band-dispersion parameters are available so far. Therefore, we restrict the discussion to the wurtzite phases, for which experimental data on, at least, the effective electron masses are available. For wz-InN, also $E_{P}$ has been determined by fitting a simplified $\mathbf{k} \cdot \mathbf{p}$ Hamiltonian to the experimental data. ${ }^{36,111}$ For wz-GaN, values for $E_{P}$ (Refs. 124 and 125) and also several reports on the effective hole masses are available. ${ }^{130}$

Wurtzite GaN. The OEPx(cLDA $)+G_{0} W_{0}$ effective electron masses in wz-GaN $\left(m_{e}^{\|}=0.19 m_{0}, m_{e}^{\perp}=0.21 m_{0}\right)$ are in very good agreement with experimental values, which scatter around $m_{e}=0.20 m_{0} \cdot{ }^{130}$ However, our calculations predict an anisotropy of the electron masses of about $10 \%$, which is larger than values found experimentally $[<1 \%-6 \%$ (Refs.
131-133)]. Our $E_{P}$ values of 17.3 and $16.3 \mathrm{eV}$ support those obtained by Rodina and Meyer ${ }^{124}(\approx 18.3$ and $\approx 17.3 \mathrm{eV})$, rather than a larger value of $E_{P} \approx 19.8 \mathrm{eV}$ reported recently by Shokhovets et al. $^{125}$

A detailed analysis of the effective hole masses has been presented by Rodina et al. ${ }^{126}$ Note that only the $A$-band masses in their work have been extracted directly from experimental data. All other effective hole masses have been calculated from the $A$-band effective masses and the spinorbit and crystal-field splitting energies within the quasicubic approximation. The effective $A$-band masses derived in the present paper $\left(m_{A}^{\|}=1.88 m_{0}\right.$ and $\left.m_{A}^{\perp}=0.33 m_{0}\right)$ agree very well with the experimental values derived by Rodina et al. $\left(m_{A}^{\|}\right.$ $=1.76 m_{0}$ and $\left.m_{A}^{\perp}=0.35 m_{0}\right)$. Adopting their values for the spin-orbit and crystal-field splitting parameters $\left(\Delta_{\mathrm{SO}}\right.$ $=0.019 \mathrm{eV} ; \Delta_{\mathrm{CR}}=0.010 \mathrm{eV}$ ), we also find a good agreement for the $B$ - and $C$-band masses (see Table VII).

Wurtzite AlN. The available experimental data on the band dispersion in wz-AIN is limited to the effective electron mass, which has been determined to be in the range of $\left(0.29-0.4 m_{0}\right){ }^{122}$ The OEPx $(\mathrm{cLDA})+G_{0} W_{0}$ values of $m_{e}^{\|}$ $=0.32 m_{0}$ and $m_{e}^{\perp}=0.33 m_{0}$ fall within this range.

Wurtzite InN. Experimentally derived effective electron masses in wz-InN scatter over a wide range (see Table VII). The most reliable seem to be those reported by $\mathrm{Wu}$ et al. ${ }^{36}$ and $\mathrm{Fu}$ and $\mathrm{Chen}^{111}$ since they explicitly account for the high carrier concentration of their samples and the nonparabolicity of the $\mathrm{CB}$ in their analysis. Their effective electron masses of $0.05 m_{0}$ (Ref. 111) and $0.07 m_{0}$ (Ref. 36) in conjunction with values for $E_{P}$ of 9.7 and $10 \mathrm{eV}$, respectively, are in good agreement with those derived from the $\mathrm{OEPx}(\mathrm{cLDA})+G_{0} W_{0} \quad$ calculations $\quad\left(m_{e}^{\|}=0.065 m_{0}, \quad m_{e}^{\perp}\right.$ $=0.068 m_{0}$ and $\left.E_{P}^{\|}=8.7 \mathrm{eV}, E_{P}^{\perp}=8.8 \mathrm{eV}\right)$. Our calculations also predict an anisotropy of the electron masses of about $5 \%$. A similar anisotropy has been reported by Hofmann et al. ${ }^{127}$ (see Table VII).

\section{Other parameter sets}

Local-density approximation. For means of comparison, we have also derived band parameters from LDA and OEPX(cLDA) calculations in the same way as for the 
(a) zb-AlN

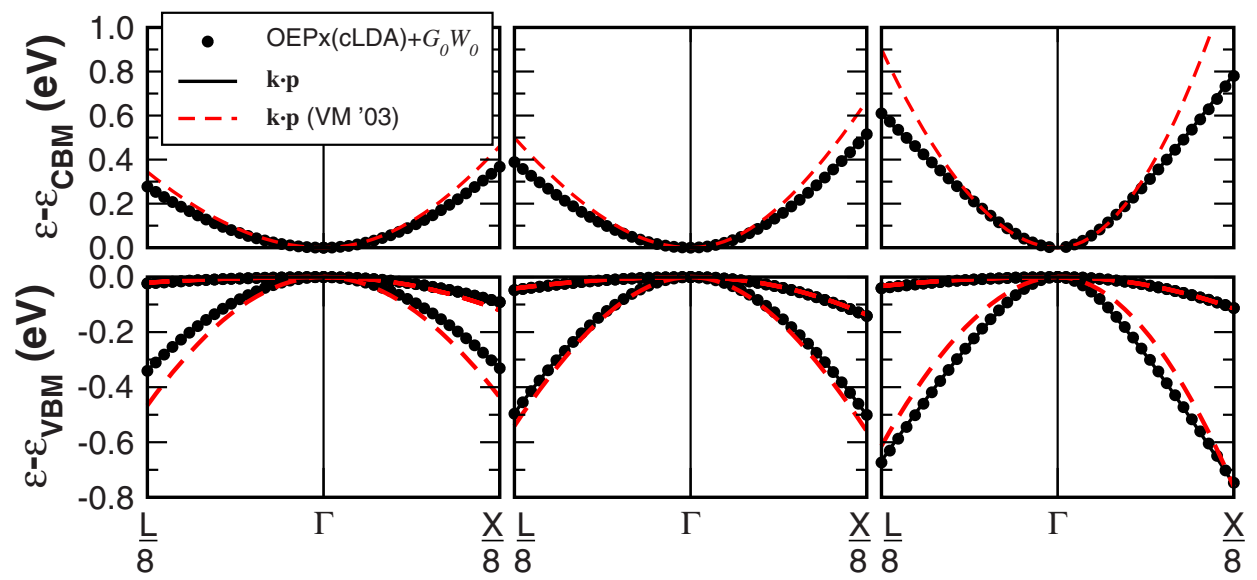

FIG. 4. (Color online) Band structure of zb-AlN (a), zb-GaN (b), and zb-InN (c) in the vicinity of $\Gamma$. The graphs show the $\operatorname{OEPx}(\mathrm{cLDA})+G_{0} W_{0}$ band structure (black circles), the corresponding $\mathbf{k} \cdot \mathbf{p}$ band structure (black solid lines), and the $\mathbf{k} \cdot \mathbf{p}$ band structure using the parameters recommended by Vurgaftman and Meyer (Refs. 26 and 116) (VM'03) (red dashed lines).
$\operatorname{OEPx}(\mathrm{cLDA})+G_{0} W_{0}$ data. LDA band structures are frequently employed for fitting parameter sets, ${ }^{118,134}$ but we will demonstrate here that the LDA is not suitable to consistently determine all parameters for the group-III nitrides accurately. The parameters derived from the LDA band structures are listed in Table VII for the wurtzite phases of GaN and AIN. Since the LDA predicts InN to be metallic, no LDA band parameters could be derived for InN.

The effective electron masses of GaN in LDA are smaller than in $\operatorname{OEPx}(\mathrm{cLDA})+G_{0} W_{0}$ and the experiment. The effective electron masses of a given material are, to a first approximation, proportional to the fundamental band gap. ${ }^{135}$ Thus, the underestimation of the effective electron masses in LDA is to some degree a natural side effect of the underestimation of the fundamental band gap. Additional factors (e.g., self-interaction) contributing to the deviation of the LDA band structure from the quasiparticle one were alluded to in Sec. II A.

The $A$-band hole masses in LDA show an increased anisotropy; the deviation from the experimental values increases. Despite the fact that the band gap of AlN is also significantly smaller in LDA than in $\operatorname{OEPx}(\mathrm{cLDA})+G_{0} W_{0}$, it is still large, i.e., well above $4 \mathrm{eV}$. Therefore, an effect on the absolute values of the effective electron masses is not visible, but the LDA predicts an anisotropy of the electron masses, with the opposite sign compared to the OEPx(cLDA) $+G_{0} W_{0}$ calculations.

OEPx(cLDA). As alluded to in Sec. II A, band gaps in the OEPx(cLDA) approach open compared to LDA (see Table III). Following the proportionallity relationship between the direct band gap and the conduction band effective mass, the latter should increase in OEPx(cLDA). This is indeed the case, as Table VII demonstrates. They are, however, also larger than the conduction-band effective masses in the $\operatorname{OEPx}(\mathrm{cLDA})+G_{0} W_{0}$ approach, despite the fact that only in InN is the OEPx(cLDA) band gap larger than in $\operatorname{OEPx}(\mathrm{cLDA})+G_{0} W_{0}$. We attribute this behavior to the approximate treatment of correlation in the $\operatorname{OEPx}(\mathrm{cLDA})$, which aversely affects the band dispersion, as explained in Sec. III D. We thus do not recommend the use of the OEPX or the OEPx(cLDA) approach alone for the determination of band parameters.
$L D A$ plus correction $(L D A+C)$. In the $\mathrm{LDA}+C$ approach, ${ }^{136}$ delta-function potentials are added at the atomic sites, which artificially push $s$-like wave functions upward in energy. As a consequence, the band gaps open due to the admixture of cation $s$ states in the conduction band. The potentials have to be fitted to available experimental data, such as the fundamental band gaps, and can be applied in an all-electron ${ }^{86,136}$ and in a pseudopotential framework. ${ }^{137}$ Carrier and Wei $\left(\mathrm{CW}^{\prime} 05\right)^{86}$ determined the effective electron and hole masses of GaN, AlN, and InN, using this method. Their results are also given in Table VII.

Their values for the effective electron masses of all three materials are in good agreement with the OEPx(cLDA) $+G_{0} W_{0}$ values. The deviations are larger for the effective hole masses. This is not too surprising because the LDA $+C$ approach predominantly affects $s$-derived bands. Since the upper valence bands around $\Gamma$ are mostly of nitrogen $2 p$ character, their description will be closer to the LDA level, whereas the conduction bands feel the additional corrections.

Empirical pseudopotential method. A semiempirical way, often used to calculate band parameters, is the EPM. ${ }^{119,120,123,129}$ In the EPM, the full atomic potentials are replaced by those of pseudoatoms, whose adjustable parameters are fitted to a set of input band parameters, typically taken from experiments. The resulting band structures can then be used analogously to fit the parameters of a $\mathbf{k} \cdot \mathbf{p}$ Hamiltonian. Since the EPM depends sensitively on the input parameters, appreciable scatter in the reported band parameters is observed (see Table $\mathrm{V}$ for a selection).

Vurgaftman and Meyer. For none of the group-III nitrides, a complete set of band parameters has so far been derived from experimental values alone. Therefore, Vurgaftman and Meyer ${ }^{26}$ compiled parameter sets comprising experimental and the most reliable theoretical values in 2003.

For wz-GaN, VM'03 recommend the experimental value of the effective electron masses of $m_{e}^{\|}=m_{e}^{\perp}=0.20 m_{0}$ and Luttinger-like parameters derived from EPM calculation by Ren et al., ${ }^{120}$ which yield effective hole masses in good agreement with experimental and the $\operatorname{OEPx}(\mathrm{cLDA})+G_{0} W_{0}$ data (see Table VII). The parameter set yields a band structure that agrees well with the $\operatorname{OEPx}(\operatorname{cLDA})+G_{0} W_{0}$ band 
TABLE VII. Band parameters of wurtzite group-III nitrides: Comparison to parameters from the literature. Listed are experimental parameters, the parameters recommended by Vurgaftman and Meyer (Ref. 26) (VM'03), parameters using the LDA and OEPx(cLDA), parameters calculated by Carrier and Wei (Ref. 86) (CW'05) using LDA+C, and values determined using the empirical pseudopotential method (EPM) by several different groups.

\begin{tabular}{|c|c|c|c|c|c|c|c|c|}
\hline & & & & & LDA & OEPx(cLDA) & & \\
\hline & Parameter & (recommended) & Expt. & $\mathrm{VM}^{\prime} 03^{\mathrm{a}}$ & (this work, & , for comparison) & $\mathrm{LDA}+\mathrm{C}^{\mathrm{b}}$ & EPM \\
\hline \multirow[t]{6}{*}{ AlN } & $m_{e}^{\|}$ & 0.32 & $0.29-0.45^{\mathrm{c}}$ & 0.32 & 0.33 & 0.38 & 0.32 & $0.23^{\mathrm{d}}, 0.24^{\mathrm{e}}, 0.27^{\mathrm{f}}$ \\
\hline & $m_{e}^{\perp}$ & 0.33 & $0.29-0.45^{\mathrm{c}}$ & 0.30 & 0.32 & 0.39 & 0.33 & $0.24^{\mathrm{d}}, 0.25^{\mathrm{e}}, 0.18^{\mathrm{f}}$ \\
\hline & $E_{P}^{\|}(\mathrm{eV})$ & 16.97 & & & 16.41 & 16.89 & & \\
\hline & $E_{P}^{\perp}(\mathrm{eV})$ & 18.17 & & & 17.45 & 17.51 & & \\
\hline & $m_{C}^{\|}$ & 3.13 & & 3.57 & 3.46 & 3.72 & 3.43 & $2.38^{\mathrm{d}}, 1.87-1.95^{\mathrm{e}}$ \\
\hline & $m_{C}^{\perp}$ & 0.69 & & 0.59 & 0.68 & 0.83 & 0.68 & $\begin{array}{c}0.49^{\mathrm{d}}, 0.43-0.48^{\mathrm{e}} \\
0.36^{\mathrm{f}}\end{array}$ \\
\hline \multirow[t]{10}{*}{$\mathrm{GaN}$} & $m_{e}^{\|}$ & 0.19 & $0.20^{\mathrm{g}}$ & 0.20 & 0.15 & 0.23 & 0.20 & $0.14^{\mathrm{d}}, 0.14^{\mathrm{e}}, 0.16^{\mathrm{f}}$ \\
\hline & $m_{e}^{\perp}$ & 0.21 & $0.20^{\mathrm{g}}$ & 0.20 & 0.17 & 0.26 & 0.22 & $0.15^{\mathrm{d}}, 0.15^{\mathrm{e}}, 0.12^{\mathrm{f}}$ \\
\hline & $E_{P}^{\|}(\mathrm{eV})$ & 17.29 & $17.8-18.7^{\mathrm{h}}, 19.8^{\mathrm{i}}$ & & 12.93 & 16.14 & & \\
\hline & $E_{P}^{\perp}(\mathrm{eV})$ & 16.27 & $16.9-17.8^{\mathrm{h}}, 19.8^{\mathrm{i}}$ & & 11.30 & 14.07 & & \\
\hline & $m_{A}^{\|}$ & 1.88 & $1.76^{\mathrm{j}}$ & 1.89 & 1.92 & 2.20 & 2.04 & $\begin{array}{c}1.89^{\mathrm{k}}, 2.37^{\mathrm{d}} \\
1.45-1.48^{\mathrm{e}}, 1.27^{\mathrm{f}}\end{array}$ \\
\hline & $m_{A}^{\perp}$ & 0.33 & $0.35^{\mathrm{j}}$ & 0.26 & 0.26 & 0.38 & 0.39 & $\begin{array}{c}0.26^{\mathrm{k}}, 0.49^{\mathrm{d}} \\
0.26-0.27^{\mathrm{e}}, 0.20^{\mathrm{f}}\end{array}$ \\
\hline & $m_{B}^{\|}$ & $0.37^{1}, 0.92^{\mathrm{m}}$ & $0.42^{\mathrm{j}}$ & & & & 0.85 & \\
\hline & $m_{B}^{\perp}$ & $0.49^{1}, 0.36^{\mathrm{m}}$ & $0.51^{\mathrm{j}}$ & & & & 0.43 & \\
\hline & $m_{C}^{\|}$ & $0.26^{1}, 0.19^{\mathrm{m}}$ & $0.30^{\mathrm{j}}$ & & & & 0.19 & \\
\hline & $m_{C}^{\perp}$ & $0.65^{1}, 1.27^{\mathrm{m}}$ & $0.68^{\mathrm{j}}$ & & & & 1.05 & \\
\hline \multirow[t]{6}{*}{$\mathrm{InN}$} & $m_{e}^{\|}$ & 0.065 & $0.07^{\mathrm{n}}, 0.05^{\mathrm{o}}, 0.04^{\mathrm{p}}, 0.085^{\mathrm{q}}$ & 0.07 & & 0.11 & 0.06 & $\begin{array}{c}0.072^{\mathrm{r}}, 0.10^{\mathrm{e}} \\
0.14^{\mathrm{f}}\end{array}$ \\
\hline & $m_{e}^{\perp}$ & 0.068 & $0.07^{\mathrm{n}}, 0.05^{\mathrm{o}}, 0.05^{\mathrm{p}}, 0.085^{\mathrm{q}}$ & 0.07 & & 0.12 & 0.07 & $\begin{array}{c}0.068^{\mathrm{r}}, 0.10^{\mathrm{e}} \\
0.10^{\mathrm{f}}\end{array}$ \\
\hline & $E_{P}^{\|}(\mathrm{eV})$ & 8.74 & $10^{\mathrm{n}}, 9.7^{\mathrm{o}}$ & & & 9.22 & & \\
\hline & $E_{P}^{\perp}(\mathrm{eV})$ & 8.81 & $10^{\mathrm{n}}, 9.7^{\mathrm{o}}$ & & & 8.67 & & \\
\hline & $m_{A}^{\|}$ & 1.81 & & 1.56 & & 2.12 & 2.09 & $\begin{array}{c}2.56-2.63^{\mathrm{r}} \\
1.35-1.43^{\mathrm{e}}, 1.56^{\mathrm{f}}\end{array}$ \\
\hline & $m_{A}^{\perp}$ & 0.13 & & 0.17 & & 0.21 & 0.14 & $\begin{array}{c}0.14-0.15^{\mathrm{r}} \\
0.18-0.20^{\mathrm{e}}, 0.17^{\mathrm{f}}\end{array}$ \\
\hline
\end{tabular}

${ }^{a}$ Vurgaftman and Meyer, Ref. 26.

${ }^{\mathrm{b}}$ Carrier and Wei, Ref. 86.

'Silveira et al., Ref. 122.

${ }^{\mathrm{d}}$ Fritsch et al., Ref. 119.

${ }^{\mathrm{e}}$ Dugdale et al., Ref. 123.

fPugh et al., Ref. 121.

${ }^{g}$ Reference 26 and references therein.

${ }^{\text {h}}$ Rodina and Meyer, Ref. 124.

iShokhovets et al., Ref. 125.

${ }^{\mathrm{j}}$ Rodina et al., Ref. 126.

${ }^{\mathrm{k}}$ Ren et al., Ref. 120.

${ }^{1}$ Calculated using $\Delta_{\mathrm{SO}}=0.019 \mathrm{eV}$ and $\Delta_{\mathrm{CR}}=0.010 \mathrm{eV}$ (Ref. 126).

${ }^{\mathrm{m} C a l c u l a t e d ~ u s i n g ~} \Delta_{\mathrm{SO}}=0.016 \mathrm{eV}$ and $\Delta_{\mathrm{CR}}=0.025 \mathrm{eV}$ (Ref. 86).

${ }^{\mathrm{n}} \mathrm{Wu}$ et al., Ref. 36.

${ }^{\circ} \mathrm{Fu}$ and Chem, Ref. 111.

pHofmann et al., Ref. 127.

Inushima et al., Ref. 128.

${ }^{\mathrm{r}}$ Fritsch et al., Ref. 129. 
structure for the $\mathrm{CB}$ and the two top VBs (see Fig. 3). It deviates, however, for the $C$ VB (the third valence band counted from the valence-band maximum), where the curvatures in the EPM band structure are too large.

Of all the compounds and phases discussed in this paper, wz-GaN is the best characterized experimentally. The good agreement between our quasiparticle band structures and those based on the parameter set recommended by VM'03 proves the quality of our $\operatorname{OEPx}(\mathrm{cLDA})+G_{0} W_{0}$ band structures.

For wz-AlN, the effective electron masses recommended by VM'03 are the averages over several theoretical values; the recommended VB parameters are theoretical values by Kim et al. ${ }^{134}$ derived from LDA calculations. These parameters yield a band structure, which is in good overall agreement with the OEPx(cLDA) $+G_{0} W_{0}$ band structure [see Fig. $3(\mathrm{a})]$. The anisotropy of the effective electron masses, however, has the opposite sign, similar to our own LDA calculations. The similarity between VM'03 (i.e., LDA) and $\operatorname{OEPx}(\mathrm{cLDA})+G_{0} W_{0}$ in the valence-band region (after adjusting $\left.\Delta_{\mathrm{CR}}\right)$ is due to the fact that in AlN valence bands are shifted rigidly compared to the LDA, as discussed in Sec. III D. In OEPx(cLDA) alone, however, the dispersion changes noticeably (similar to what was observed for $\mathrm{InN}$, see Fig. 1) giving rise to appreciably different band parameters (Table VII).

For wz-InN, VM'03 recommend the experimental effective electron masses by Wu et al. ${ }^{36}\left(m_{e}^{\|}=m_{e}^{\perp}=0.07 m_{0}\right)$ and the EPM values from Pugh et al. ${ }^{121}$ for the VB. The pseudopotentials used by Pugh et al. were designed to reproduce their LDA calculations, which had been "scissor corrected" to the incorrect band gap of $2.0 \mathrm{eV}$. These parameters are therefore to no avail from today's perspective.

\section{CONCLUSION}

We have derived consistent and unbiased band parameters for the wurtzite and zinc-blende phases of GaN, AlN, and InN from accurate OEPx $(\mathrm{cLDA})+G_{0} W_{0}$ band structure calculations. The band parameters are in very good agreement with the available experimental data, proving the reliability of the method. We also provide reliable values for those parameters, which have not been determined experimentally, e.g., the band parameters of the zinc-blende phases of $\mathrm{GaN}$, AlN, and InN or the $E_{p}$ and VB parameters of wurtzite phases. These parameters are essential for understanding the physics of these materials. We have derived complete and consistent parameter sets for the description of the band structures of the group-III nitrides within the $\mathbf{k} \cdot \mathbf{p}$ theory. The $\mathbf{k} \cdot \mathbf{p}$ method is widely used for modeling and simulating (opto-)electronic devices. The parameters presented in this work overcome the apparent lack of consistent band parameter sets for such simulations.

Finally, we remark that the combination of the $\mathbf{k} \cdot \mathbf{p}$ with the $G_{0} W_{0}$ method is not restricted to the $4 \times 4(8 \times 8) \mathbf{k} \cdot \mathbf{p}$ Hamiltonians discussed in this work. Since we expect $G_{0} W_{0}$ to provide the same accuracy for the whole Brillouin zone, the parameters for more complex Hamiltonians can be fitted in the same way.

\section{ACKNOWLEDGMENTS}

We would like to acknowledge fruitful discussions with Axel Hoffmann, Peter Kratzer, Martin Fuchs, Christoph Freysoldt, and Chris G. Van de Walle. This work was in part funded by the Volkswagen Stiftung/Germany, the DFG through SFB 296, SFB 787, and the EU's sixth framework program through the NANOQUANTA (NMP4-CT-2004500198) and SANDiE (NMP4-CT-2004-500101) networks of excellence.

\section{APPENDIX A: $\mathbf{k} \cdot \mathbf{p}$ HAMILTONIAN}

The $\mathbf{k} \cdot \mathbf{p}$ Hamiltonian used in the present work is based on the one introduced in Ref. 11 for zinc-blende crystals and its extension to wurtzite crystal structures in Refs. 14, 117, 123, and 148. It will be described in the following.

Neglecting spin-orbit interactions, the $8 \times 8$ Hamilton matrix reduces to $4 \times 4$ and can be decomposed into two separate matrices:

$$
H=H_{1}+H_{2} \text {. }
$$

The matrix $H_{1}$ represents the pure $4 \times 4 \mathbf{k} \cdot \mathbf{p}$ description of the conduction and valence bands neglecting all remote band contributions. For wurtzite crystals, it is given by

$$
=\left(\begin{array}{cccc}
H_{1} & & & \\
\widetilde{E}_{g}+\Delta_{\mathrm{CR}}+\frac{\hbar^{2} k^{2}}{2 m_{0}} & i P_{2} k_{x} & i P_{2} k_{y} & i P_{1} k_{z} \\
-i P_{2} k_{x} & \Delta_{\mathrm{CR}}+\frac{\hbar^{2} k^{2}}{2 m_{0}} & 0 & 0 \\
-i P_{2} k_{y} & 0 & \Delta_{\mathrm{CR}}+\frac{\hbar^{2} k^{2}}{2 m_{0}} & 0 \\
-i P_{1} k_{z} & 0 & 0 & \frac{\hbar^{2} k^{2}}{2 m_{0}}
\end{array}\right) .
$$

Here, $m_{0}$ is the free electron mass. $\widetilde{E}_{g}$ is identical to the fundamental band gap $E_{g}$ for all materials with a positive crystal-field splitting $\Delta_{\mathrm{CR}}$, i.e., GaN and InN, and $E_{g}+\left|\Delta_{\mathrm{CR}}\right|$ for materials with negative $\Delta_{\mathrm{CR}}$, i.e., AlN. The parameters $P_{1 / 2}$ are proportional to the absolute value of the $\mathrm{CB} / \mathrm{VB}$ dipole matrix elements at $\Gamma$. They are customarily expressed in terms of the Kane parameters $E_{P 1 / 2}$ :

$$
P_{1 / 2}=\sqrt{\frac{\hbar^{2}}{2 m_{0}} E_{P 1 / 2}}
$$

For zinc-blende crystals, $H_{1}$ simplifies through $P_{1}=P_{2}\left(E_{P 1}\right.$ $\left.=E_{P 2}\right)$ and $\Delta_{\mathrm{CR}}=0$.

The matrix $\mathrm{H}_{2}$ describes the influences of all bands not considered explicitly by the $4 \times 4$ method. For wurtzite crystals, it is defined by 


$$
H_{2}=\left(\begin{array}{cccc}
A_{2}^{\prime}\left(k_{x}^{2}+k_{y}^{2}\right)+A_{1}^{\prime} k_{z}^{2} & B_{2} k_{y} k_{z} & B_{2} k_{x} k_{z} & B_{1} k_{x} k_{z} \\
B_{2} k_{y} k_{z} & L_{1}^{\prime} k_{x}^{2}+M_{1} k_{y}^{2}+M_{2} k_{z}^{2} & N_{1}^{\prime} k_{x} k_{y} & N_{2}^{\prime} k_{x} k_{z}-N_{3}^{\prime} k_{x} \\
B_{2} k_{x} k_{z} & N_{1}^{\prime} k_{y} k_{x} & M_{1} k_{x}^{2}+L_{1}^{\prime} k_{y}^{2}+M_{2} k_{z}^{2} & N_{2}^{\prime} k_{y} k_{z}-N_{3}^{\prime} k_{y} \\
B_{1} k_{x} k_{z} & N_{2}^{\prime} k_{z} k_{x}+N_{3}^{\prime} k_{x} & N_{2}^{\prime} k_{z} k_{x}+N_{3}^{\prime} k_{y} & M_{3}\left(k_{x}^{2}+k_{y}^{2}\right)+L_{2}^{\prime} k_{z}^{2}
\end{array}\right) .
$$

The parameters in $\mathrm{H}_{2}$ are defined in Ref. 11 in terms of optical matrix elements for zinc-blende crystals. The corresponding definitions for wurtzite crystals can be found in, e.g., Ref. 117. The parameters are related to the more commonly used effective electron masses, $m_{e}^{\|}$and $m_{e}^{\perp}$, and Luttinger-like parameters, $A_{i}$, by

$$
\begin{gathered}
A_{1}^{\prime}=\frac{\hbar^{2}}{2}\left(\frac{1}{m_{e}^{\|}}-\frac{1}{m_{0}}\right)-\frac{P_{1}^{2}}{E_{g}}, \\
A_{2}^{\prime}=\frac{\hbar^{2}}{2}\left(\frac{1}{m_{e}^{\perp}}-\frac{1}{m_{0}}\right)-\frac{P_{2}^{2}}{E_{g}}, \\
L_{1}^{\prime}=\frac{\hbar^{2}}{2 m_{0}}\left(A_{2}+A_{4}+A_{5}-1\right)+\frac{P_{1}^{2}}{E_{g}}, \\
L_{2}^{\prime}=\frac{\hbar^{2}}{2 m_{0}}\left(A_{1}-1\right)+\frac{P_{2}^{2}}{E_{g}}, \\
M_{1}=\frac{\hbar^{2}}{2 m_{0}}\left(A_{2}+A_{4}-A_{5}-1\right), \\
M_{2}=\frac{\hbar^{2}}{2 m_{0}}\left(A_{1}+A_{3}-1\right), \\
M_{3}=\frac{\hbar^{2}}{2 m_{0}}\left(A_{2}-1\right), \\
N_{1}^{\prime}=\frac{\hbar^{2}}{2 m_{0}} 2 A_{5}+\frac{P_{1}^{2}}{E_{g}}, \\
N_{2}^{\prime}=\frac{\hbar^{2}}{2 m_{0}} \sqrt{2} A_{6}+\frac{P_{1} P_{2}}{E_{g}}, \\
N_{3}^{\prime}=i \sqrt{2} A_{7} .
\end{gathered}
$$

The corresponding relations for zinc-blende crystals are

$$
\begin{gathered}
\left(A_{1}^{\prime}=A_{2}^{\prime}=\right) A^{\prime}=\frac{\hbar^{2}}{2}\left(\frac{1}{m_{e}}-\frac{1}{m_{0}}\right)-\frac{P^{2}}{E_{g}}, \\
\left(L_{1}^{\prime}=L_{2}^{\prime}=\right) L^{\prime}=-\frac{\hbar^{2}}{2 m_{0}}\left(\gamma_{1}+4 \gamma_{2}\right)+\frac{P^{2}}{E_{g}}, \\
\left(M_{1}=M_{2}=M_{3}=\right) M=-\frac{\hbar^{2}}{2 m_{0}}\left(\gamma_{1}-2 \gamma_{2}\right),
\end{gathered}
$$

$$
\begin{gathered}
\left(N_{1}^{\prime}=N_{2}^{\prime}=\right) N^{\prime}=-\frac{\hbar^{2}}{2 m_{0}} 6 \gamma_{3}+\frac{P^{2}}{E_{g}}, \\
N_{3}^{\prime}=0 .
\end{gathered}
$$

Here, $m_{e}$ denotes the electron effective mass and $\gamma_{i}$ the Luttinger parameters.

The parameters $B_{1 / 2}$ occur due to the lack of inversion symmetry in zinc-blende and wurtzite crystals. Their inclusion in the k $\cdot \mathbf{p}$ Hamiltonian does not yield a noticeable improvement of the fitted results. Therefore, they have been omitted throughout this work.

\section{APPENDIX B: EFFECTIVE HOLE MASSES}

In detail, the equations, connecting the effective hole masses to the Luttinger(-like) parameters, are (for wurtzite crystals) ${ }^{117}$

$$
\begin{gathered}
m_{0} / m_{A}^{\|}=-\left(A_{1}+A_{3}\right), \\
m_{0} / m_{A}^{\perp}=-\left(A_{2}+A_{4}\right), \\
m_{0} / m_{B}^{\|}=-\left[A_{1}+\left(\frac{E_{B}}{E_{B}-E_{C}}\right) A_{3}\right], \\
m_{0} / m_{B}^{\perp}=-\left[A_{2}+\left(\frac{E_{B}}{E_{B}-E_{C}}\right) A_{4}\right], \\
m_{0} / m_{C}^{\|}=-\left[A_{1}+\left(\frac{E_{C}}{E_{C}-E_{B}}\right) A_{3}\right], \\
m_{0} / m_{C}^{\perp}=-\left[A_{2}+\left(\frac{E_{C}}{E_{C}-E_{B}}\right) A_{4}\right],
\end{gathered}
$$

with

$$
\begin{aligned}
& E_{B}=\frac{\Delta_{\mathrm{CR}}-\Delta_{\mathrm{SO}} / 3}{2}+\sqrt{\left(\frac{\Delta_{\mathrm{CR}}-\Delta_{\mathrm{SO}} / 3}{2}\right)^{2}+2\left(\frac{\Delta_{\mathrm{SO}} / 3}{2}\right)^{2}}, \\
& E_{C}=\frac{\Delta_{\mathrm{CR}}-\Delta_{\mathrm{SO}} / 3}{2}-\sqrt{\left(\frac{\Delta_{\mathrm{CR}}-\Delta_{\mathrm{SO}} / 3}{2}\right)^{2}+2\left(\frac{\Delta_{\mathrm{SO}} / 3}{2}\right)^{2}} .
\end{aligned}
$$

For AlN, the indices $\mathrm{A}, \mathrm{B}$, and $\mathrm{C}$ have to be interchanged: $\mathrm{A} \rightarrow \mathrm{B}, \mathrm{B} \rightarrow \mathrm{C}$, and $\mathrm{C} \rightarrow \mathrm{A}$. For zinc-blende crystals, it follows that ${ }^{27}$

$$
m_{0} / m_{\mathrm{hh}}^{[001]}=\gamma_{1}-2 \gamma_{2},
$$




$$
\begin{gathered}
m_{0} / m_{\mathrm{hh}}^{[110]}=\frac{1}{2}\left(2 \gamma_{1}-\gamma_{2}-3 \gamma_{3}\right), \\
m_{0} / m_{\mathrm{hh}}^{[111]}=\gamma_{1}-2 \gamma_{3}, \\
m_{0} / m_{\mathrm{lh}}^{[001]}=\gamma_{1}+2 \gamma_{2},
\end{gathered}
$$

$$
\begin{gathered}
m_{0} / m_{\mathrm{lh}}^{[110]}=\frac{1}{2}\left(2 \gamma_{1}+\gamma_{2}+3 \gamma_{3}\right), \\
m_{0} / m_{\mathrm{lh}}^{[111]}=\gamma_{1}+2 \gamma_{3}, \\
m_{0} / m_{\mathrm{so}}=\gamma_{1}-\frac{E_{P} \Delta_{\mathrm{SO}}}{3 E_{g}\left(E_{g}+\Delta_{\mathrm{SO}}\right)} .
\end{gathered}
$$

*rinke@fhi-berlin.mpg.de

${ }^{1}$ T. M. S. Nakamura and M. Senoh, Jpn. J. Appl. Phys., Part 2 30, L1998 (1991).

${ }^{2}$ S. Nakamura, Science 281, 956 (1998).

${ }^{3}$ S. Nakamura, M. Senoh, S. Nagahama, N. Iwasa, T. Yamada, T. Matsushita, Y. Sugimoto, and H. Kiyoku, Appl. Phys. Lett. 69, 4056 (1996).

${ }^{4}$ S. Nakamura, M. Senoh, S. Nagahama, N. Iwasa, T. Yamada, T. Matsushita, H. Kiyoku, and Y. Sugimoto, Jpn. J. Appl. Phys., Part 2 35, L74 (1996).

${ }^{5}$ Y. Taniyasu, M. Kasu, and T. Makimoto, Nature (London) 441, 325 (2006).

${ }^{6}$ V. Adivarahan, W. H. Sun, A. Chitnis, M. Shatalov, S. Wu, H. P. Maruska, and M. A. Khan, Appl. Phys. Lett. 85, 2175 (2004).

${ }^{7}$ M. Stutzmann, G. Steinhoff, M. Eickhoff, O. Ambacher, C. E. Nebela, J. Schalwig, R. Neuberger, and G. Müller, Diamond Relat. Mater. 11, 886 (2002).

${ }^{8}$ J. Schalwig, G. Müller, M. Eickhoff, O. Ambacher, and M. Stutzmann, Mater. Sci. Eng., B B93, 207 (2002).

${ }^{9}$ H. Lu, W. J. Schaff, and L. F. Eastman, J. Appl. Phys. 96, 3577 (2004).

${ }^{10}$ S. Kako, C. Santori, K. Hoshino, S. Götzinger, Y. Yamamoto, and Y. Arakawa, Nat. Mater. 5, 887 (2006).

${ }^{11}$ E. O. Kane, in Band Theory and Transport Properties, Handbook on Semiconductors Vol. 1, edited by W. Paul (North-Holland, Amsterdam, 1982), p. 195.

${ }^{12}$ A. D. Andreev and E. P. O'Reilly, Phys. Rev. B 62, 15851 (2000).

${ }^{13}$ V. A. Fonoberov and A. A. Balandin, J. Appl. Phys. 94, 7178 (2003).

${ }^{14}$ M. Winkelnkemper, A. Schliwa, and D. Bimberg, Phys. Rev. B 74, 155322 (2006).

${ }^{15}$ J. C. Slater and G. F. Koster, Phys. Rev. 94, 1498 (1954).

${ }^{16}$ T. Saito and Y. Arakawa, Physica E (Amsterdam) 15, 169 (2002).

${ }^{17}$ V. Ranjan, G. Allan, C. Priester, and C. Delerue, Phys. Rev. B 68, 115305 (2003).

${ }^{18}$ S. Schulz, S. Schumacher, and G. Czycholl, Phys. Rev. B 73, 245327 (2006)

${ }^{19}$ L. W. Wang and A. Zunger, J. Chem. Phys. 100, 2394 (1994).

${ }^{20}$ L. Hedin, Phys. Rev. 139, A796 (1965).

${ }^{21}$ W. G. Aulbur, L. Jönsson, and J. W. Wilkins, Solid State Phys. 54, 1 (2000).

${ }^{22}$ G. Onida, L. Reining, and A. Rubio, Rev. Mod. Phys. 74, 601 (2002).

${ }^{23}$ P. Rinke, A. Qteish, J. Neugebauer, C. Freysoldt, and M. Scheffler, New J. Phys. 7, 126 (2005).

${ }^{24}$ G. Bastard and J. A. Brum, IEEE J. Quantum Electron. 22, 1625
(1986).

${ }^{25}$ W. Walukiewicz, J. W. Ager III, K. M. Yu, Z. Lilienthal-Weber, J. Wu, S. X. Li, R. E. Jones, and J. D. Denlinger, J. Phys. D 39, R83 (2006).

${ }^{26}$ I. Vurgaftman and J. R. Meyer, J. Appl. Phys. 94, 3675 (2003).

${ }^{27}$ I. Vurgaftman and J. Meyer, J. Appl. Phys. 89, 5815 (2001).

${ }^{28}$ V. Y. Davydov et al., Phys. Status Solidi B 229, R1 (2002).

${ }^{29}$ J. Wu, W. Walukiewicz, K. M. Yu, J. W. Ager III, E. E. Haller, H. Lu, W. Schaff, Y. Saiton, and Y. Nanishi, Appl. Phys. Lett. 80, 3967 (2002).

${ }^{30}$ Y. Nanishi, Y. Saito, and T. Yamaguchi, Jpn. J. Appl. Phys., Part 1 42, 2549 (2003).

${ }^{31}$ A. Sher, M. van Schilfgaarde, M. A. Berding, S. Krishnamurthy, and A.-B. Chen, MRS Internet J. Nitride Semicond. Res. 4S1, G5.1 (1999).

${ }^{32}$ F. Bechstedt and J. Furthmüller, J. Cryst. Growth 246, 315 (2002).

${ }^{33}$ J. G. Lozano, F. M. Morales, R. García, D. González, V. Lebedev, C. Y. Wang, V. Cimalla, and O. Ambacher, Appl. Phys. Lett. 90, 091901 (2007).

${ }^{34}$ P. Rinke, A. Qteish, M. Winkelnkemper, D. Bimberg, J. Neugebauer, and M. Scheffler, Appl. Phys. Lett. 89, 161919 (2006).

${ }^{35}$ P. Rinke, A. Qteish, J. Neugebauer, and M. Scheffler, $\boldsymbol{\Psi}_{\mathbf{k}}$ newsletter highlight Vol. 79 (2007), p. 163 [Phys. Status Solidi B (to be published)].

${ }^{36}$ J. Wu, W. Walukiewicz, W. Shan, K. M. Yu, J. W. Ager III, E. E. Haller, H. Lu, and W. J. Schaff, Phys. Rev. B 66, 201403(R) (2002).

${ }^{37}$ The issue of self-consistency in $G W$ is still a matter of debate (Refs. 138-142). Unlike in DFT, a self-consistent solution of the full set of equations for the self-energy in many-body perturbation theory would go beyond the $G W$ approximation and successively introduce higher order electron-electron interactions with every iteration step. Solving the $G W$ equations self-consistently is therefore inconsistent if no higher order electron-electron interactions are included. It was first observed for the homogeneous electron gas (Ref. 143) that the spectral features broaden with increasing number of iterations in the self-consistency cycle. Similarly, for closed shell atoms, the good agreement with experiment for the ionization energy after the first iteration is lost upon iterating the equations to self-consistency (Ref. 140). Imposing self-consistency in an approximate fashion (Refs. 142 and 144-146) is not unique, and different methods yield different results. Since the controversies regarding self-consistency within $G W$ have not been resolved conclusively yet, we refrain from any self-consistent treatment and remain with the zeroth order in the self-energy $\left(G_{0} W_{0}\right)$. 
${ }^{38}$ M. Rohlfing, P. Krüger, and J. Pollmann, Phys. Rev. Lett. 75, 3489 (1995).

${ }^{39}$ M. Rohlfing, P. Krüger, and J. Pollmann, Phys. Rev. B 57, 6485 (1998).

${ }^{40}$ Z. Sitar, M. J. Paisley, J. Ruan, J. W. Choyke, and R. F. Davis, J. Mater. Sci. Lett. 11, 261 (1992).

${ }^{41}$ H. Okumura, S. Yoshida, and T. Okahisa, Appl. Phys. Lett. 64, 2997 (1994).

${ }^{42}$ G. Ramirez-Flores, H. Navarro-Contreras, A. Lastras-Martínez, R. C. Powell, and J. E. Greene, Phys. Rev. B 50, 8433 (1994).

${ }^{43}$ M. Usuda, N. Hamada, K. Shiraishi, and A. Oshiyama, Jpn. J. Appl. Phys., Part 2 43, L407 (2004).

${ }^{44}$ T. Kotani and M. van Schilfgaarde, Solid State Commun. 121, 461 (2002).

${ }^{45}$ A. Qteish, A. I. Al-Sharif, M. Fuchs, M. Scheffler, S. Boeck, and J. Neugebauer, Phys. Rev. B 72, 155317 (2005).

${ }^{46}$ A. Qteish, P. Rinke, M. Scheffler, and J. Neugebauer, Phys. Rev. B 74, 245208 (2006).

${ }^{47}$ Contrary to the OEPx approach, the $G W$ method is not free from self-interaction. A small amount of self-screening is reintroduced by the correlation part of the self-energy, which is, however, significantly smaller than the self-interaction error of the LDA (Ref. 147).

${ }^{48}$ W. Walukiewicz, S. X. Li, J. Wu, K. M. Yu, J. W. Ager III, E. E. Haller, H. Lu, and W. Schaff, J. Cryst. Growth 269, 119 (2004).

${ }^{49}$ The lattice parameters of wz-InN and wz-GaN have been slightly refined compared to our recently published calculations (Ref. 34). The influences of the adjustment on the different band parameters will be indicated where necessary.

${ }^{50}$ http://www.sfhingx.de

${ }^{51}$ H. N. Rojas, R. W. Godby, and R. J. Needs, Phys. Rev. Lett. 74, 1827 (1995).

${ }^{52}$ M. M. Rieger, L. Steinbeck, I. White, H. Rojas, and R. Godby, Comput. Phys. Commun. 117, 211 (1999).

${ }^{53}$ L. Steinbeck, A. Rubio, L. Reining, M. Torrent, I. White, and R. Godby, Comput. Phys. Commun. 125, 105 (2000).

${ }^{54}$ C. Freysoldt, P. Eggert, P. Rinke, A. Schindlmayr, R. W. Godby, and M. Scheffler, Comput. Phys. Commun. 176, 1 (2007).

${ }^{55}$ J. P. Perdew and A. Zunger, Phys. Rev. B 23, 5048 (1981).

${ }^{56}$ D. M. Ceperley and B. J. Alder, Phys. Rev. Lett. 45, 566 (1980).

${ }^{57}$ M. Moukara, M. Städele, J. A. Majewski, P. Vogl, and A. Görling, J. Phys.: Condens. Matter 12, 6783 (2000).

${ }^{58}$ A. J. Morris, M. Stankovski, K. T. Delaney, P. Rinke, P. GarcíaGonzález, and R. W. Godby, Phys. Rev. B 76, 155106 (2007).

${ }^{59}$ M. Städele, M. Moukara, J. A. Majewski, P. Vogl, and A. Görling, Phys. Rev. B 59, 10031 (1999).

${ }^{60}$ D. Chandrasekhar, D. J. Smith, S. Strite, M. E. Lin, and H. Morkoc, J. Cryst. Growth 152, 135 (1995).

${ }^{61}$ V. Cimalla, J. Pezoldt, G. Ecke, R. Kosiba, O. Ambacher, L. Spieß, H. Lu, W. J. Schaff, and G. Teichert, Appl. Phys. Lett. 83, 3468 (2003).

${ }^{62}$ A. P. Lima, A. Tabata, J. R. Leite, S. Kaiser, D. Schikora, B. Schöttker, T. Frey, D. J. As, and K. Lischka, J. Cryst. Growth 201/202, 396 (1999).

${ }^{63}$ I. Petrov, E. Mojab, R. C. Powell, J. E. Greene, L. Hultman, and J.-E. Sundgren, Appl. Phys. Lett. 60, 2491 (1992).

${ }^{64}$ M. P. Thompson, G. W. Auner, T. S. Zheleva, K. A. Jones, S. J. Simko, and J. N. Hilfiker, J. Appl. Phys. 89, 3331 (2001).

${ }^{65}$ T. Lei, M. Fanciulli, R. J. Molnar, T. D. Moustakas, R. J. Graham, and J. Scanlon, Appl. Phys. Lett. 59, 944 (1991).
${ }^{66}$ T. Lei, T. D. Moustakas, R. J. Graham, Y. He, and S. J. Berkowitz, J. Appl. Phys. 71, 4933 (1992).

${ }^{67}$ B. Maleyre, S. Ruffenach, O. Briot, and A. van der Lee, Superlattices Microstruct. 36, 527 (2004).

${ }^{68}$ S. Strite and H. Morkoç, J. Vac. Sci. Technol. B 10, 1237 (1992).

${ }^{69}$ H. Schulz and K. H. Thiermann, Solid State Commun. 23, 815 (1977).

${ }^{70}$ W. M. Yim, E. J. Stofko, P. J. Zanzucchi, J. I. Pankove, M. Ettenberg, and S. L. Gilbert, J. Appl. Phys. 44, 292 (1972).

${ }^{71}$ H. P. Maruska and J. J. Tietjen, Appl. Phys. Lett. 15, 327 (1969).

${ }^{72}$ L. Chen et al., Appl. Phys. Lett. 85, 4334 (2004).

${ }^{73}$ P. B. Perry and R. F. Rutz, Appl. Phys. Lett. 33, 319 (1978).

${ }^{74}$ Q. Guo and A. Yoshida, Jpn. J. Appl. Phys., Part 1 33, 2453 (1994).

${ }^{75}$ H. Akamaru, A. Onodera, T. Endo, and O. Mishima, J. Phys. Chem. Solids 63, 887 (2002).

${ }^{76}$ J. Chen, W. Z. Shen, H. Ogawa, and Q. X. Guo, Appl. Phys. Lett. 84, 4866 (2004).

${ }^{77}$ J. Li, K. B. Nam, M. L. Nakarmi, J. Y. Lin, H. X. Jiang, P. Carrier, and S.-H. Wei, Appl. Phys. Lett. 83, 5163 (2003).

${ }^{78}$ J. Schörmann, D. J. As, K. Lischka, P. Schley, R. Goldhahn, S. F. Li, W. Löffler, M. Hetterich, and H. Kalt, Appl. Phys. Lett. 89, 261903 (2006).

${ }^{79}$ J. Bhattacharyya, S. Ghosh, M. R. Gokhale, B. M. Arora, H. Lu, and W. J. Schaff, Appl. Phys. Lett. 89, 151910 (2006).

${ }^{80}$ M. Higashiwaki and T. Matsui, J. Cryst. Growth 251, 494 (2003).

${ }^{81}$ M. Losurdo, G. Bruno, T.-H. Kim, S. Choi, and A. Brown, Appl. Phys. Lett. 88, 121928 (2006).

${ }^{82}$ R. Goldhahn, P. Schley, A. Winzer, M. Rakel, C. Cobet, N. Esser, H. Lu, and W. Schaff, J. Cryst. Growth 288, 273 (2006).

${ }^{83}$ Throughout this work, $\Delta_{\mathrm{CR}}>0$ refers to the $\Gamma_{9}-\Gamma_{7}-\Gamma_{7} \mathrm{VB}$ odering and $\Delta_{\mathrm{CR}}<0$ to $\Gamma_{7}-\Gamma_{9}-\Gamma_{7}$.

${ }^{84} \mathrm{As}$ a result of the adjusted lattice parameters, the wz-InN band gap undergoes a slight correction from 0.72 to $0.69 \mathrm{eV}$ in the present paper compared to Ref. 34 .

${ }^{85} \mathrm{As}$ in the case of wz-InN, the band gap of wz-GaN is slightly smaller (by $0.08 \mathrm{eV}$ ) for the current set of lattice parameters compared to the value published in Ref. 34.

${ }^{86}$ P. Carrier and S.-H. Wei, J. Appl. Phys. 97, 033707 (2005).

${ }^{87}$ J. J. A. Freitas, G. C. B. Braga, E. Silveira, J. G. Tischler, and M. Fatemi, Appl. Phys. Lett. 83, 2584 (2003).

${ }^{88}$ B. Gil, O. Briot, and R.-L. Aulombard, Phys. Rev. B 52, R17028 (1995).

${ }^{89}$ B. Gil, F. Hamdani, and H. Morkoç, Phys. Rev. B 54, 7678 (1996).

${ }^{90}$ S.-H. Wei and A. Zunger, Appl. Phys. Lett. 69, 2719 (1996).

${ }^{91}$ A. Janotti and C. G. Van de Walle, Phys. Rev. B 75, 121201(R) (2007).

${ }^{92}$ H. Schulz and K. H. Thiemann, Solid State Commun. 23, 815 (1977).

${ }^{93}$ H. Xia, Q. Xia, and A. L. Ruoff, Phys. Rev. B 47, 12925 (1993).

${ }^{94}$ M. Ueno, M. Yoshida, A. Onodera, O. Shimomura, and K. Takemura, Phys. Rev. B 49, 14 (1994).

${ }^{95}$ Numerical Data and Functional Relationships in Science and Technology, Landolt-Börnstein, New Series, Group III, Vol. 22, Pt. A, edited by O. Madelung and M. Schulz (Springer, Berlin, 1987).

${ }^{96}$ P. Perlin, I. Gorczyca, N. E. Christensen, I. Grzegory, H. Teisseyre, and T. Suski, Phys. Rev. B 45, 13307 (1992).

${ }^{97}$ D. L. Camphausen and G. A. N. Connell, J. Appl. Phys. 42, 4438 
(1971).

${ }^{98}$ W. Shan, T. J. Schmidt, R. J. Hauenstein, J. J. Song, and B. Goldenberg, Appl. Phys. Lett. 66, 3492 (1995).

${ }^{99}$ S. Kim, I. P. Herman, J. A. Tuchman, K. Doverspike, L. B. Rowland, and D. K. Gaskill, Appl. Phys. Lett. 67, 380 (1995).

${ }^{100}$ P. Perlin, L. Mattos, N. A. Shapiro, J. Kruger, W. S. Wong, T. Sands, N. W. Cheung, and E. R. Weber, J. Appl. Phys. 85, 2385 (1999).

${ }^{101}$ K. Reimann, M. Steube, O. Brandt, H. Yang, and K. H. Ploog, J. Appl. Phys. 84, 2971 (1998).

${ }^{102}$ G. Franssen et al., Appl. Phys. Lett. 89, 121915 (2006).

${ }^{103}$ S. X. Li, J. Wu, E. E. Haller, W. Walukiewicz, W. Shan, H. Lu, and W. Schaff, Appl. Phys. Lett. 83, 4963 (2003).

${ }^{104}$ Q. Xia, H. Xia, and A. L. Ruoff, J. Appl. Phys. 73, 8198 (1993).

${ }^{105}$ T. Mashimo, M. Uchino, A. N. T. Kobayashi, E. Takasawa, T. S. Y. Noguchi, H. Hikosaka, K. Fukuoka, and Y. Syono, J. Appl. Phys. 86, 6710 (1999).

${ }^{106}$ M. Ueno, A. Onodera, O. Shimomura, and K. Takemura, Phys. Rev. B 45, R10123 (1992).

${ }^{107}$ S. J. Hwang, W. Shan, R. J. Hauenstein, J. J. Song, M.-E. Lin, S. Strite, B. N. Sverdlov, and H. Morkoç, Appl. Phys. Lett. 64, 2928 (1994).

${ }^{108}$ Z. X. Liu, A. R. G. Ni, K. Syassen, H. Siegle, C. Thomsen, B. Schöttker, D. J. As, and D. Schikora, J. Appl. Phys. 86, 929 (1999).

${ }^{109}$ S.-H. Wei and A. Zunger, Phys. Rev. B 60, 5404 (1999).

${ }^{110}$ O. Gunnarsson and B. I. Lundqvist, Phys. Rev. B 13, 4274 (1976).

${ }^{111}$ S. P. Fu and Y. F. Chen, Appl. Phys. Lett. 85, 1523 (2004).

${ }^{112}$ D. Gershoni, C. Henry, and G. A. Baraff, IEEE J. Quantum Electron. 29, 2433 (1993).

${ }^{113}$ O. Stier, M. Grundmann, and D. Bimberg, Phys. Rev. B 59, 5688 (1999).

${ }^{114}$ S. Hackenbuchner, J. Majewski, G. Zandler, and P. Vogl, J. Cryst. Growth 230, 607 (2001).

${ }^{115}$ K. H. Lee, J. H. Na, R. A. Taylor, S. N. Yi, S. Birner, Y. S. Park, C. M. Park, and T. W. Kang, Appl. Phys. Lett. 89, 023103 (2006).

${ }^{116}$ For the plots, we used the effective electron masses and $\gamma_{i}\left(A_{i}\right)$ parameters recommended in Ref. 26 and the band gaps (and crystal-field splitting energies) from our $\operatorname{OEPx}(\mathrm{cLDA})+G_{0} W_{0}$ calculations.

${ }^{117}$ S. L. Chuang and C. S. Chang, Phys. Rev. B 54, 2491 (1996).

${ }^{118}$ M. Suzuki, T. Uenoyama, and A. Yanase, Phys. Rev. B 52, 8132 (1995).

${ }^{119}$ D. Fritsch, H. Schmidt, and M. Grundmann, Phys. Rev. B 67, 235205 (2003).

${ }^{120}$ G. B. Ren, Y. M. Liu, and P. Blood, Appl. Phys. Lett. 74, 1117 (1999).

${ }^{121}$ S. K. Pugh, D. J. Dugdale, S. Brand, and R. A. Abram, Semi- cond. Sci. Technol. 14, 23 (1999).

${ }^{122}$ E. Silveira, J. J. A. Freitas, M. Kneissl, D. W. Treat, N. M. Johnson, G. A. Slack, and L. J. Schowalter, Appl. Phys. Lett. 84, 3501 (2004).

${ }^{123}$ D. J. Dugdale, S. Brand, and R. A. Abram, Phys. Rev. B 61, 12933 (2000).

${ }^{124}$ A. V. Rodina and B. K. Meyer, Phys. Rev. B 64, 245209 (2001).

${ }^{125}$ S. Shokhovets, G. Gobsch, and O. Ambacher, Appl. Phys. Lett. 86, 161908 (2005).

${ }^{126}$ A. V. Rodina, M. Dietrich, A. Göldner, L. Eckey, A. Hoffmann, A. L. Efros, M. Rosen, and B. K. Meyer, Phys. Rev. B 64, 115204 (2001).

${ }^{127}$ A. T. Hofmann, T. Chavdarov, V. Darakchieva, H. Lu, W. J. Schaff, and M. Schubert, Phys. Status Solidi C 3, 1854 (2006).

${ }^{128}$ T. Inushima, M. Higashiwaki, and T. Matsui, Phys. Rev. B 68, 235204 (2003).

${ }^{129}$ D. Fritsch, H. Schmidt, and M. Grundmann, Phys. Rev. B 69, 165204 (2004).

${ }^{130}$ See Ref. 26 and references therein.

${ }^{131}$ P. Perlin et al., Appl. Phys. Lett. 68, 1114 (1996).

${ }^{132}$ A. Kasic, M. Schubert, S. Einfeldt, D. Hommel, and T. E. Tiwald, Phys. Rev. B 62, 7365 (2000).

${ }^{133}$ B. K. Meyer, D. Volm, A. Graber, H. C. Alt, T. Detchprohm, A. Amano, and I. Akasaki, Solid State Commun. 95, 597 (1995).

${ }^{134}$ K. Kim, W. R. L. Lambrecht, B. Segall, and M. van Schilfgaarde, Phys. Rev. B 56, 7363 (1997).

${ }^{135}$ B. R. Nag, Phys. Status Solidi B 237, R1 (2003).

${ }^{136}$ N. E. Christensen, Phys. Rev. B 30, 5753 (1984).

${ }^{137}$ D. Segev, A. Janotti, and C. G. Van de Walle, Phys. Rev. B 75, 035201 (2007).

${ }^{138}$ W. Ku and A. G. Eguiluz, Phys. Rev. Lett. 89, 126401 (2002).

${ }^{139}$ M. L. Tiago, S. Ismail-Beigi, and S. G. Louie, Phys. Rev. B 69, 125212 (2004).

${ }^{140}$ K. Delaney, P. García-González, A. Rubio, P. Rinke, and R. W. Godby, Phys. Rev. Lett. 93, 249701 (2004).

${ }^{141}$ N. E. Zein, S. Y. Savrasov, and G. Kotliar, Phys. Rev. Lett. 96, 226403 (2006)

${ }^{142}$ M. van Schilfgaarde, T. Kotani, and S. Faleev, Phys. Rev. Lett. 96, 226402 (2006).

${ }^{143}$ B. Holm and U. von Barth, Phys. Rev. B 57, 2108 (1998).

${ }^{144}$ W. Luo, S. Ismail-Beigi, M. L. Cohen, and S. G. Louie, Phys. Rev. B 66, 195215 (2002)

${ }^{145}$ A. Fleszar and W. Hanke, Phys. Rev. B 71, 045207 (2005).

${ }^{146}$ M. Marsili, O. Pulci, F. Bechstedt, and R. Del Sole, Phys. Rev. B 72, 115415 (2005).

${ }^{147}$ W. Nelson, P. Bokes, P. Rinke, and R. W. Godby, Phys. Rev. A 75, 032505 (2007).

${ }^{148}$ G. Bir and G. Pikus, Symmetry and Strain-Induced Effects in Semiconductors (Halsted, New York, 1974). 